\title{
OPERATOR HOLES AND EXTENSIONS OF SECTORIAL OPERATORS AND DUAL PAIRS OF CONTRACTIONS
}

\author{
MARK MALAMUD
}

\begin{abstract}
A description of the set of $m$-sectorial extensions of a dual pair $\left\{A_{1}, A_{2}\right\}$ of nonnegative operators is obtained. Some classes of nonaccretive extensions of the dual pair $\left\{A_{1}, A_{2}\right\}$ are described too. Both problems are reduced to similar problems for a dual pair $\left\{T_{1}, T_{2}\right\}$ of nondensely defined symmetric contractions $T_{j}=\left(I-A_{j}\right)\left(I+A_{j}\right)^{-1}, j \in\{1,2\}$. In turn these problems are reduced to the investigation of the corresponding operator "holes". A complete description of the set of all proper and improper extensions of a nonnegative operator is obtained too.
\end{abstract}

\section{INTRODUCTION}

In the theory of extensions of a nonnegative operator $A\left(\subset A^{*}\right)$ in a Hilbert space $\mathfrak{H}$ to a selfadjoint or $m$-sectorial 23] operator there are two well-known approaches in which extensions $\widetilde{A} \supset A$ in various classes are described in diverse forms. One of these, proposed by M. G. Krein in [25] (see also [1, 34]) uses the linear fractional transformation $T_{1}=$ $(I-A)(I+A)^{-1}$ to reduce the problem to the description of various classes of extensions $T \supset T_{1}$ of a nondensely defined (on the subspace $\mathfrak{H}_{1}=(I+A) \mathfrak{H}$ ) symmetric contraction $T_{1}$.

The other approach to the description of proper extensions $\widetilde{A}$ of an operator $A>0$ was proposed by Vishik [39] and Birman 99. They associate with each extension $\widetilde{A} \supset A$ (not necessarily selfadjoint) a "boundary" operator $B$ acting in an auxiliary space $\mathcal{H}(\operatorname{dim} \mathcal{H}=$ $\left.\operatorname{dim}\left(A^{*}-i\right) \mathfrak{H}\right)$, and they describe the properties of the extension $\widetilde{A}=\widetilde{A}_{B}$ in terms of the operator $B$, i.e. essentially in terms of the boundary conditions if $A$ is a differential operator. This approach was subsequently formalized in the concept of a "boundary triplet' and was developed in later papers by many authors (see for instance [19, 13] and and references therein).

We remark that the methods used in these approaches are essentially different, as are the descriptions obtained with their help.

Recall that a closed densely defined operator $A$ in $\mathfrak{H}$ is called sectorial with a half-angle $\varphi \in(0, \pi / 2]$ if

$$
\operatorname{Re}(A f, f) \geq \cot \varphi \cdot|\operatorname{Im}(A f, f)|, \quad f \in \operatorname{dom} A .
$$

It is called a maximal sectorial ( $m$-sectorial) and is put in class $S_{\mathfrak{H}}(\varphi)$ if additionally $\rho(A) \neq \emptyset$. If $\varphi=\pi / 2$ inequality (1.1) turns into the inequality $\operatorname{Re}(A f, f) \geq 0$ and the class $S_{\mathfrak{H}}(\pi / 2)$ is the class of maximal accretive operators. Denote also by $S_{\mathfrak{H}}(0)$ the class of nonnegative selfadjoint operators in $\mathfrak{H}$ and note that $S_{\mathfrak{H}}(0)=\cap_{\varphi>0} S_{\mathfrak{H}}(\varphi)$.

1991 Mathematics Subject Classification. Primary 47A57, 47B25; Secondary 47A55, 47B65.

Key words and phrases. Completion, shorted operator, generalized Schur complement, selfadjoint contractive extension, nonnegative selfadjoint extension, Friedrichs and Kreĭn-von Neumann extension. 
In this paper we solve among others the following two problems.

Problem 1S. Given a closed nonnegative symmetric operator $A \geq 0$ in $\mathfrak{H}$. Describe the set $\operatorname{Ext}_{A}(\varphi)$ of all proper and improper $S_{\mathfrak{H}}(\varphi)$-extensions of $A$ with $\varphi \in[0, \pi / 2]$.

Problem 2S. Given a dual pair $\left\{A_{1}, A_{2}\right\}$ of closed nonnegative symmetric operators in $\mathfrak{H}$. Find necessary and sufficient conditions for $\left\{A_{1}, A_{2}\right\}$ to admit an extension $\widetilde{A}\left(A_{1} \subset \widetilde{A} \subset A_{2}^{*}\right)$ of the class $S_{\mathfrak{H}}(\varphi)$ with $\varphi \in[0, \pi / 2]$ and describe the set $\operatorname{Ext}_{\left\{A_{1}, A_{2}\right\}}(\varphi)$ of such extensions.

Note, that Problem $1 \mathrm{~S}$ is solvable for any $\varphi \in[0, \pi / 2]$. Indeed, it is known (see [22, 1, 34]) that any symmetric operator $A \geq 0$ admits a selfadjoint extension $\widetilde{A} \geq 0$, say the Friedrichs extension $A_{F}$. In other words, $\operatorname{Ext}_{A}(0) \neq \emptyset$, hence $\operatorname{Ext}_{A}(\varphi) \neq \emptyset$ for any $\varphi \in(0, \pi / 2]$.

A complete description of the set Ext ${ }_{A}(0)$ in terms of "boundary" operators have been obtained in [9] in the case of a positive definite operator $A$. The set $\operatorname{Extp}_{A}(\varphi):=\operatorname{Ext}_{\{A, A\}}(\varphi)$, $\varphi \in[0, \pi / 2]$, of all proper $m$-sectorial extensions of an operator $A \geq 0$ with zero lower bound was described via boundary triplets and Weyl functions in 24] and 15]. Another description in the framework of Krein's approach has been obtained in [5, 6].

On the other hand, even a solvability criterion of Problem $2 \mathrm{~S}$ was unknown. We will show below that Problem $2 \mathrm{~S}$ is not necessary solvable for any $\varphi \in[0, \pi / 2)$. It may even happen that it is solvable only with $\varphi=\pi / 2$.

We will also discuss the following more general problems.

Problem 3S. Given a sectorial operator $A$ with a half-angle $\varphi_{0} \in[0, \pi / 2)$. Describe the set $\operatorname{Ext}_{A}(\varphi)$ of all $S_{\mathfrak{H}}(\varphi)$-extensions of $A$ with $\varphi \geq \varphi_{0}$.

Problem 4S. Given a dual pair $\left\{A_{1}, A_{2}\right\}$ of sectorial operators in $\mathfrak{H}$. Find necessary and sufficient conditions for $\left\{A_{1}, A_{2}\right\}$ to have an extension $\widetilde{A}\left(A_{1} \subset \widetilde{A} \subset A_{2}^{*}\right)$ belonging to the class $S_{\mathfrak{H}}(\varphi)$ with $\varphi \geq \varphi_{0}$ and describe the set $\operatorname{Ext}_{\left\{A_{1}, A_{2}\right\}}(\varphi)$ of all such extensions.

By the Kato-Schechter theorem (see [23]) any sectorial operator $A$ obeying (1.1) with $\varphi_{0} \in(0, \pi / 2)$ admits $m$-sectorial extension, say the Friedrichs extension $A_{F}$. In other words, $\operatorname{Ext}_{A}\left(\varphi_{0}\right) \neq \emptyset$, hence $\operatorname{Ext}_{A}(\varphi) \neq \emptyset$ for $\varphi \geq \varphi_{0}$, Thus, Problem 3S is solvable for any $\varphi \geq \varphi_{0}$.

Note, that even a criterion of solvability of Problem $4 \mathrm{~S}$ is unknown.

In accordance with Krein's approach we consider a linear fractional transformation $T_{1}=$ $(I-A)(I+A)^{-1}$ of a sectorial operator $A$. It is clear that $T_{1}$ is a nondensely defined contraction, $T_{1}\left(\in\left[\mathfrak{H}_{1}, \mathfrak{H}\right]\right)$, obeying the following condition

$$
\left\|T_{1} \sin \varphi \pm i \cos \varphi \cdot I\right\| \leq 1 \quad \operatorname{dom} T_{1}=\mathfrak{H}_{1}:=\operatorname{ran}(I+A) .
$$

We put an operator $T$ in the class $C_{\mathfrak{H}}(\varphi)$ with $\varphi \in(0, \pi / 2]$ if $\operatorname{dom} T=\mathfrak{H}$ and inequality (1.2) holds with $T$ in place of $T_{1}$. Note that $C_{\mathfrak{H}}(\pi / 2)$ is the class of all contractions in $\mathfrak{H}$ and denote by $C_{\mathfrak{H}}(0)$ the class of all selfadjoint contractions in $\mathfrak{H}$.

Now we can reformulate Problems $1 \mathrm{~S}-4 \mathrm{~S}$ in the following way.

Problem 1C. Given a nondendely defined symmetric operator $T_{1} \in\left[\mathfrak{H}_{1}, \mathfrak{H}\right]$. Describe the set of all proper and improper $C_{\mathfrak{H}}(\varphi)$-extensions of $T$ with $\varphi \geq \varphi_{0}$.

Problem 2C. Given a dual pair $\left\{T_{1}, T_{2}\right\}$ of nondensely defined symmetric contractions. Find necessary and sufficient conditions for $\left\{T_{1}, T_{2}\right\}$ to admit an extension $T \in C_{\mathfrak{H}}(\varphi)$ with $\varphi \geq \varphi_{0}$ and describe the set $\operatorname{Ext}_{\left\{T_{1}, T_{2}\right\}}(\varphi)$ of all such extensions.

Problem 3C. Given a nondendely defined operator $T_{1} \in\left[\mathfrak{H}_{1}, \mathfrak{H}\right]$ obeying (1.2) with $\varphi=\varphi_{0}$. Describe the set $\operatorname{Ext}_{T_{1}}(\varphi)$ of all $C_{\mathfrak{H}}(\varphi)$-extensions of $T$ with $\varphi \geq \varphi_{0}$.

Problem 4C. Given a dual pair $\left\{T_{1}, T_{2}\right\}$ of nondensely defined contractions, obeying condition (1.2) with $\varphi=\varphi_{0}(\in[0, \pi / 2])$. Find necessary and sufficient conditions for $\left\{T_{1}, T_{2}\right\}$ 
to have an extension $T \in C_{\mathfrak{H}}(\varphi)$ with $\varphi \geq \varphi_{0}$ and describe the set $\operatorname{Ext}_{\left\{T_{1}, T_{2}\right\}}(\varphi)$ of all such extensions.

It is convenient to regard Problems $1 \mathrm{C}$ and $3 \mathrm{C}$ as a problem on the "completion" of a contractive operator matrix $T_{1}=\left(\begin{array}{l}T_{11} \\ T_{21}\end{array}\right)$ to form a matrix $T=\left(T_{j k}\right)_{j, k=1}^{2}$ which is connected in a natural way with the problem of extending of a dual pair of contractions to operators in various classes. A description is given in terms of operator balls, "holes", and objects close to them.

Starting point of our investigation is a description of the set of all contractive extensions of a dual pair of contractions $\left\{T_{1}=\left(\begin{array}{l}T_{11} \\ T_{21}\end{array}\right), T_{2}=\left(\begin{array}{l}T_{11}^{*} \\ T_{12}^{*}\end{array}\right)\right\}$ or what is the same a description of all "completions" of a matrix

$$
T_{0}=\left(\begin{array}{cc}
T_{11} & T_{12} \\
T_{21} & *
\end{array}\right)=\left(\begin{array}{cc}
T_{11} & D_{T_{11}^{*}} U \\
V D_{T_{11}} & *
\end{array}\right)
$$

to form a contractive matrix $T=\left(T_{i j}\right)_{i, j=1}^{2}$.

It has been shown in [7, 11, 12, 38] that all missing blocks $T_{22}$ in (1.3) form an operator ball $B\left(-V T_{11}^{*} U ; D_{V^{*}}, D_{U}\right)$ :

$$
T_{22}=-V T_{11}^{*} U+D_{V^{*}} K D_{U}, \quad\|K\| \leq 1 .
$$

Our approach to Problems $1 \mathrm{C}-4 \mathrm{C}$ is essentially based on the solution to the following

Problem 5. Given two operator balls

$$
B\left(C_{ \pm} ; R_{l}^{ \pm}, R_{r}^{ \pm}\right)=\left\{Z \in[\mathcal{H}]: Z=C_{ \pm}+R_{l}^{ \pm} K R_{r}^{ \pm}, \quad\|K\| \leq 1\right\} .
$$

Find a criterion for an operator "hole" ("loone")

$$
L:=B\left(C_{+} ; R_{l}^{+}, R_{r}^{+}\right) \cap B\left(C_{-} ; R_{l}^{-}, R_{r}^{+}\right)
$$

to be nonempty and obtain a parametrization of $L$.

Problem 5 naturally arrises in diferent areas and is of interest itself. We will show here that all Problems 1C-4C are reduced to Problem 5. Analysis of operator holes (1.5) corresponding to Problems $1 \mathrm{C}-4 \mathrm{C}$ shows that degree of difficulty of any Problem jC with $j \in\{1, \ldots, 4\}$, can be characterized by means of the corresponding radii $R_{l}^{ \pm}$and $R_{r}^{ \pm}$. From this point of view Problem 2C with $T_{1}=T_{2}\left(\Longleftrightarrow T_{11}=T_{11}^{*}, T_{21}=T_{12}^{*}\right)$ is the simplest one. It is reduced to Problem 5 with four equal radii $R_{l}^{ \pm}=R_{r}^{ \pm}=D_{U}$. This problem is always solvable and it is equivalent to a description of the $\operatorname{set} \operatorname{Extp}_{T_{1}}(\varphi)$ of proper $C(\varphi)$-extensions of a symmetric contraction $T_{1}$, which has been solved in [5, 6] by different method.

Next, a solution to Problem $2 \mathrm{C}$ is equivalent to a description of missing blocks $T_{22}$ in matrix (1.3) (with $T_{11}=T_{11}^{*}$ ) such that $T=\left(T_{i j}\right) \in C_{\mathfrak{H}}(\varphi)$, that is $T \sin \varphi \pm i \cos \varphi \cdot I \in C_{\mathfrak{H}}(\pi / 2)$. Due to (1.4) this problem is reduced to Problem 5 with $R_{l}^{+}=R_{l}^{-}=D_{V^{*}}$ and $R_{r}^{+}=R_{r}^{-}=D_{U}$. It is not always solvable in general (see below).

Further, Problem 3C with $\varphi_{0}>0$ is reduced to Problem 5 (see [30, and Remark 3.18) with different left radii $R_{l}^{+} \neq R_{l}^{-}$and equal right radii $R_{r}^{+}=R_{r}^{-}$, while it is always solvable.

Finally, the most difficult Problem $4 \mathrm{C}$ with $\varphi_{0}>0$ is reduced to Problem 5 with different left radii $R_{l}^{+} \neq R_{l}^{-}$and different right radii $R_{r}^{+} \neq R_{r}^{-}$(see Proposition 3.17).

I don't know a criterion of solvability of Problem 5 if either $R_{l}^{+} \neq R_{l}^{-}$or $R_{r}^{+} \neq R_{r}^{-}$, while a parametrization of the hole $L$ can be easily obtained if at least one of its elements is known (see [24, 30]). However a solution to Problem 5 with $R_{l}^{+}=R_{l}^{-}$and $R_{r}^{+}=R_{r}^{-}$is rather simple and is contained in Lemma 3.3 . 
The paper is organized as follows.

In Section 2 we summarize some definitions and statements which are necessary in the sequel.

In Section 3 we present a solution to Problem 2C (see Theorem 3.4) based on Lemma 3.3 on a parametrization of an operator hole (1.5) with $R_{l}^{+}=R_{l}^{-}$and $R_{r}^{+}=R_{r}^{-}$. It is worth to note that though $\operatorname{Ext}_{\left\{T_{1}, T_{2}\right\}}(\pi / 2) \neq \emptyset$, it may happen that $\operatorname{Ext}_{\left\{T_{1}, T_{2}\right\}}(\varphi)=\emptyset$ for any $\varphi \in\left[\varphi_{0}, \pi / 2\right)$. The solvability of Problem $2 \mathrm{C}$ depends on the operator

$$
Q_{0}=D_{V}^{-1}(I-V U) D_{U}^{-1}
$$

More precisely, $\operatorname{Ext}_{\left\{T_{1}, T_{2}\right\}}(\varphi) \neq \emptyset$ if and only if $\varphi \in\left[\varphi_{1}, \pi / 2\right]$ where $\varphi_{1}=\arccos \left(\left\|Q_{0}\right\|^{-1}\right)$. In particular, Ext $\operatorname{ET}_{\left.1, T_{2}\right\}}(\varphi)=\emptyset$ for any $\varphi \in(0, \pi / 2)$ if and only if $Q_{0}$ is unbounded.

Further, in Section 3 we present a description of the set $\operatorname{Ext}_{T_{1}}(\varphi)$ of all (proper and improper) extensions of a symmetric contraction $T_{1}\left(\in\left[\mathfrak{H}_{1}, \mathfrak{H}\right]\right)$ (see Theorem [3.14). This result gives a complete solution to Problem 1C.

We also present here (see Propositions 3.6]and 3.8) a partial description of the set $\operatorname{Ext}_{\left\{T_{1}, T_{2}\right\}}^{e}(\varphi)$ of extreme points of the set $\operatorname{Ext}_{\left\{T_{1}, T_{2}\right\}}(\varphi)$. It is interesting to note that even in a finite dimensional case $(\operatorname{dim} \mathfrak{H}=n<\infty)$ the set $C_{\mathfrak{H}}^{e}(\varphi)$ of extreme points of the operator loone $C_{\mathfrak{H}}(\varphi)$ with $\varphi \in(0, \pi / 2)$ essentially differs from the set $C_{\mathfrak{H}}^{e}(\pi / 2)$ of extreme points of the operator ball in $\mathbb{C}^{n}$. Namely, though the set $C_{\mathfrak{H}}^{e}(\pi / 2)$ consists of unitary matrices, the set $C_{\mathfrak{H}}^{e}(\varphi), \varphi \in(0, \pi / 2)$, in addition to normal matrices with "boundary spectrum" contains continuum nonnormal matrices with "nonboundary" spectrum.

Finally, in Proposition 3.17. we discuss a reduction of Problem 4C to Problem 5.

In Section 4 we investigate noncontractive extensions of a dual pair $\left\{T_{1}, T_{2}\right\}$ of symmetric contractions. Namely, we consider a (not necessary contractive) extension $T_{K}$ of the form (1.3), (1.4) and calculate the Schur complement of any of the operators

$$
G^{ \pm}:=\left(G_{i j}^{ \pm}\right)_{i, j=1}^{2}:=I-T_{K} T_{K}^{*} \pm i \cot \varphi\left(T_{K}-T_{K}^{*}\right), \quad \varphi \in(0, \pi / 2] .
$$

More precisely, assuming (for simplicity) that $0 \in \rho\left(G_{11}\right)$ we prove (see Theorem 4.1) the following identities

$$
\sin ^{2} \varphi \cdot\left[G_{22}^{ \pm}-G_{21}^{ \pm} G_{11}^{-1} G_{12}^{ \pm}\right]=D_{U} \cdot\left[I-\left(K^{*} \sin \varphi \mp i Q^{*}\right)(K \sin \varphi \pm i Q)\right] \cdot D_{U},
$$

where $Q$ is the closure of $Q_{0}$.

Using (1.6) we describe the classes $C_{\mathfrak{H}}\left(\varphi ; \varkappa^{ \pm}\right)$of operators $T_{K}$ obeying conditions $\operatorname{dim} \operatorname{ran}\left(G^{ \pm}\right)_{-}=\varkappa^{ \pm}$, where $\varkappa^{ \pm} \in \mathbb{Z}_{+}$and $G_{-}$stands for the "negative" part of the operator $G=G^{*}$. Some applications of this result to the boundary value problems can be found in [29]. Moreover, formula (1.6) makes it possible to give another solution to Problem $2 \mathrm{C}$ as well as to obtain some complements to Theorem 3.4 .

In Section 5 we investigate completions of an incomplete matrix $T_{0}^{\prime}=\left(\begin{array}{cc}T_{11} & * \\ 0 & T_{22}\end{array}\right)$. Namely, in Proposition 5.2 we describe the set of some classes of noncontractive completions of $T_{0}^{\prime}$. This result complements and generalizes the result of Nagy and Foias [35].

Moreover, in Proposition [5.5] we describe the sets of $C_{\mathfrak{H}}(\varphi)$-completions of $T_{0}^{\prime}$, giving an answer to Yu. L. Shmul'yan's question. This description is given in terms of operator holes.

Some results of the paper have been announced in [28] and partially published (with proofs) in 24. 
Notations. By $\mathfrak{H}$ and $\mathcal{H}$ we denote separable Hilbert spaces; $\left[\mathfrak{H}_{1}, \mathfrak{H}_{2}\right]$ stands for the set of all bounded linear operators from $\mathfrak{H}_{1}$ to $\mathfrak{H}_{2} ;[\mathfrak{H}]:=[\mathfrak{H}, \mathfrak{H}] ; \mathcal{C}(\mathfrak{H})$ stands for the set of closed operators in $\mathfrak{H}$. We denote by $\rho(T), \sigma(T)$ and $\sigma_{p p}(T)$ the resolvent set, the spectrum and the purely point spectrum of $T(\in \mathcal{C}(\mathfrak{H}))$ respectively; $\sigma_{p}(T)$ stands for the set of eigenvalues of $T$; $\operatorname{dom} T$ and $\operatorname{ran} T$ stand for the domain of definition and the range of the operator $T$ respectively. As usual $E_{T}(\cdot)$ stands for the spectral measure (resolution of the identity) of a self-adjoint operator $T \in \mathcal{C}(\mathfrak{H}) ; T_{-}:=T E_{T}(0, \infty)$.

\section{Preliminaries}

2.1. Dual pairs of contractions. We recall a definition of a dual pair of bounded operators.

Definition 2.1. Let $\mathfrak{H}=\mathfrak{H}_{1} \oplus \mathfrak{H}_{2}=\mathfrak{H}_{1}^{\prime} \oplus \mathfrak{H}_{2}^{\prime}$ be orthogonal decompositions of the Hilbert space $H$. Operators $T_{1} \in\left[\mathfrak{H}_{1}, \mathfrak{H}\right], T_{2} \in\left[\mathfrak{H}_{1}^{\prime}, \mathfrak{H}\right]$ are said to form a dual pair of bounded operators if

$$
\left(T_{1} f, g\right)=\left(f, T_{2} g\right), \quad f \in \mathfrak{H}_{1}, g \in \mathfrak{H}_{1}^{\prime} .
$$

An operator $T(\in[\mathfrak{H}])$ is termed an extension of the dual pair $\left\{T_{1}, T_{2}\right\}$ if

$$
T\left\lceil\mathfrak { H } _ { 1 } = T _ { 1 } \quad \text { and } \quad T ^ { * } \left\lceil\mathfrak{H}_{2}=T_{2} .\right.\right.
$$

The set of all extensions of a dual pair $\left\{T_{1}, T_{2}\right\}$ is denoted by $\operatorname{Ext}_{\left\{T_{1}, T_{2}\right\}}$.

When rewritten in the block-matrix representation with respect to the pointed out decompositions of the space $\mathfrak{H}$, the operators $T_{1}$ and $T_{2}$ form a dual pair if and only if

$$
T_{1}=\left(\begin{array}{c}
T_{11} \\
T_{21}
\end{array}\right), \quad T_{2}=\left(\begin{array}{c}
T_{11}^{*} \\
T_{21}^{\prime}
\end{array}\right)
$$

with $T_{11} \in\left[\mathfrak{H}_{1}, \mathfrak{H}_{1}^{\prime}\right], T_{21} \in\left[\mathfrak{H}_{1}, \mathfrak{H}_{2}^{\prime}\right], T_{21}^{\prime} \in\left[\mathfrak{H}_{1}^{\prime}, \mathfrak{H}_{2}\right]$.

Setting $T_{12}=\left(T_{21}^{\prime}\right)^{*}$, an extension $T$ of the DP $\left\{T_{1}, T_{2}\right\}$ can be rewritten in the form

$$
T=\left(\begin{array}{ll}
T_{11} & T_{12} \\
T_{21} & T_{22}
\end{array}\right) \quad \text { with } \quad T_{22} \in\left[\mathfrak{H}_{2}, \mathfrak{H}_{2}^{\prime}\right]
$$

In this case the problem of description of a certain class $X$ of extensions of the dual pair $\left\{T_{1}, T_{2}\right\}$ is equivalent to the problem of completing an incomplete block-matrix $\left(\begin{array}{cc}T_{11} & T_{12} \\ T_{21} & *\end{array}\right)$ with respect to the matrix $T$ of the form (2.3) and such that $T \in X$.

In what follows we consider contractive extensions of a dual pair of contractions $\left\{T_{1}, T_{2}\right\}$. The union of all such extensions will be denoted by $\operatorname{Ext}_{\left\{T_{1}, T_{2}\right\}}(\pi / 2)$.

The set $\operatorname{Ext}_{\left\{T_{1}, T_{2}\right\}}(\pi / 2)$ turns out to be an operator ball in the sence of the following definition.

Definition 2.2. The totality of the operators $Z \in[\mathfrak{H}]$ of the form

$$
Z=C_{0}+R_{l} K R_{r}, \quad\|K\| \leq 1
$$

is referred to as an operator ball $B\left(C_{0} ; R_{l}, R_{r}\right)$.

Here $C_{0}$ is called the center of the ball, and $R_{l}=R_{l}^{*} \geq 0$ and $R_{r}=R_{r}^{*} \geq 0$ are called left and right radii respectively.

We will use the following simple and known result. 
Lemma 2.3. 18] Let $Q_{j} \in[\mathfrak{H}], j \in\{1,2,3\}, Q_{3}=Q_{3}^{*}, Q_{1}>0$ and $0 \in \rho\left(Q_{1}\right)$. Then the iequality

$$
Z^{*} Q_{1} Z+Z^{*} Q_{2}+Q_{2}^{*} Z+Q_{3} \leq 0
$$

has a solution if and only if

$$
Q_{2}^{*} Q_{1}^{-1} Q_{2}-Q_{3} \geq 0
$$

Under this condition the set of the solutions of the inequality (2.5) makes up an operator ball $B\left(C_{0} ; R_{l} ; R_{r}\right)$ of the form (2.4) with

$$
C_{0}=-Q_{1}^{-1} Q_{2}, \quad R_{l}=Q_{1}^{-1 / 2} \quad \text { and } \quad R_{r}=\left(Q_{2}^{*} Q_{1}^{-1} Q_{2}-Q_{3}\right)^{1 / 2} .
$$

2.2. The operators $T_{1}$ and $T_{2}$ of the form (2.2) are contractive if and only if

$$
\begin{aligned}
& T_{11}^{*} T_{11}+T_{21}^{*} T_{21} \leq I \Longleftrightarrow T_{21}^{*} T_{21} \leq D_{T_{11}}:=I-T_{11}^{*} T_{11}, \\
& T_{11} T_{11}^{*}+T_{12} T_{12}^{*} \leq I \Longleftrightarrow T_{12} T_{12}^{*} \leq D_{T_{11}^{*}}:=I-T_{11} T_{11}^{*} .
\end{aligned}
$$

It is known (and it is obvious) that these relations are equivalent to the following ones

$$
T_{21}=V D_{T_{11}}, \quad T_{12}=D_{T_{11}^{*}} U
$$

with contractions $V$ and $U\left(V \in\left[\mathfrak{H}_{1}, \mathfrak{H}_{2}^{\prime}\right], U \in\left[\mathfrak{H}_{2}, \mathfrak{H}_{1}^{\prime}\right]\right)$, which are uniquelly determined provided that ker $V \supset \operatorname{ker} D_{T_{11}}$ and ker $U^{*} \supset \operatorname{ker} D_{T_{11}^{*}}$, that is $V^{*}=D_{T_{11}}^{-1} T_{21}^{*}$ and $U=D_{T_{11}^{*}}^{-1} T_{12}$. A complete description of the set $\operatorname{Ext}_{\left\{T_{1}, T_{2}\right\}}(\pi / 2)$ is contained in the following theorem. We will essentially use it in the sequel.

Theorem 2.4. (7], 11, 12, 38]). Let $\left\{T_{1}, T_{2}\right\}$ be a dual pair of contractions,

$$
T_{1}=\left(\begin{array}{c}
T_{11} \\
T_{21}
\end{array}\right)=\left(\begin{array}{c}
T_{11} \\
V D_{T_{11}}
\end{array}\right), \quad T_{2}=\left(\begin{array}{c}
T_{11}^{*} \\
T_{12}
\end{array}\right)=\left(\begin{array}{c}
T_{11}^{*} \\
U^{*} D_{T_{11}^{*}}
\end{array}\right),
$$

$\mathcal{H}_{1}=\overline{\operatorname{ran}}\left(D_{U}\right)$ and $\mathcal{H}_{2}=\overline{\operatorname{ran}}\left(D_{V^{*}}\right)$. Then the formula

$$
T:=T_{K}=\left(\begin{array}{cc}
T_{11} & D_{T_{11}^{*}} U \\
V D_{T_{11}} & T_{22}
\end{array}\right), \quad T_{22}=-V T_{11}^{*} U+D_{V^{*}} K D_{U}
$$

establishes a bijective correspondence between all contractive extensions $T:=T_{K}=\left(T_{i j}\right) \in$ $\operatorname{Ext}_{\left\{T_{1}, T_{2}\right\}}(\pi / 2)$ and all contractions $K \in\left[\mathcal{H}_{1}, \mathcal{H}_{2}\right]$.

Thus, the set $\operatorname{Ext}{ }_{\left\{T_{1}, T_{2}\right\}}(\pi / 2)$ forms an operator ball $B\left(C_{0} ; R_{l}, R_{r}\right)$ with the center $C_{0}=$ $-V T_{11}^{*} U$ and left and right radii $R_{l}=D_{V^{*}}$ and $R_{r}=D_{U}$ respectively.

Remark 2.5. Let us make some historical remarks concerning Theorem 2.4. The case $T_{11}=T_{11}^{*}, T_{12}=T_{21}^{*}$ was considered by M.G. Krein [25] in connection with selfadjoint extensions of positive unbounded operators while his description of the class $\operatorname{Ext}_{\left\{T_{1}, T_{1}\right\}}(0)$ differs from that followed from Theorem 2.4. The existence of contractive extensions of a DPC $\left\{T_{1}, T_{2}\right\}$ (that is the fact $\operatorname{Ext}_{\left\{T_{1}, T_{2}\right\}}(\pi / 2) \neq \varnothing$ ) was first established by B.S. Nagy and C. Foias [36, p.190, by means of a corresponding generalization of the Krein's method [25]. Note also that the claim $\operatorname{Ext}_{\left\{T_{1}, T_{2}\right\}}(\pi / 2) \neq \varnothing$ is implicitly contained in [32, 33]. Another proof of the existence part of Theorem 2.4 has also been obtained by S. Parrot 31.

The complete description of the set $\operatorname{Ext}_{\left\{T_{1}, T_{2}\right\}}(\pi / 2)$, i.e., Theorem 2.4, was obtained in [7, 11, 12, 38. In the special case $\left(T_{21}=0\right)$ Theorem [2.4 has been obtained by B.Sz.-Nagy and C.Foias [35] much earlier. Several other proofs of Theorem [2.4, based on different ideas, 
can also be found in [17, 24, 30]. In particular the proof of C. Foias and A.E. Frazho [17] is based on Redheffer's products, the author's proof in 30] is based on Lemma 2.3.

\subsection{Extreme points of the unit ball.}

Recall the following

Definition 2.6. Let $G$ be a closed convex set in a Banach space $X$. A point $f \in G$ is called an extreme point of $G$ if it does not admit a represetation $f=f_{1}+(1-t) f_{2}$ with $f_{1}, f_{2} \in G, f_{1} \neq f_{2}$ and $t \in(0,1)$.

Let $\mathfrak{H}_{1}, \mathfrak{H}_{2}$ be Hilbert spaces. An operator $T\left(\in\left[\mathfrak{H}_{1}, \mathfrak{H}_{2}\right]\right)$ is called a partial isometry if $T^{*} T=P$, where $P$ is an orthoprojection in $\mathfrak{H}_{1}$. An operator $T\left(\in\left[\mathfrak{H}_{1}, \mathfrak{H}_{2}\right]\right)$ is a maximal partial isometry if either $T$ or $T^{*}$ is an isometry from $\mathfrak{H}_{1}$ to $\mathfrak{H}_{2}$, that is, if either $T^{*} T=I_{\mathfrak{H}_{1}}$ or $T T^{*}=I_{\mathfrak{H}_{2}}$.

In the sequel we need the following result which is well known in the case $\mathfrak{H}_{1}=\mathfrak{H}_{2}$ (see [20]). The general case can be easily derived from the known one.

Proposition 2.7. The set $\mathfrak{R}_{1}^{e}$ of extreme points of the unit ball $\mathfrak{R}_{1}:=\left\{T: T \in\left[\mathfrak{H}_{1}, \mathfrak{H}_{2}\right],\|T\| \leq\right.$ 1) in $\left[\mathfrak{H}_{1}, \mathfrak{H}_{2}\right]$ consists of maximal partial isometries from $\mathfrak{H}_{1}$ to $\mathfrak{H}_{2}$.

\subsection{Sectorial operators and $C(\varphi)$-contractions.}

Definition 2.8. 23]. A closed linear operator $A$ in a Hilbert space $\mathfrak{H}$ is called sectorial with vertex zero and half-angle $\varphi \in(0 ; \pi / 2)$ if its numerical range is contained in sector $G_{\varphi}=\{z \in \mathbb{C}:|\arg z| \leq \varphi<\pi / 2\}$, that is

$$
\cot \varphi \cdot|\operatorname{Im}(A f, f)| \leq \operatorname{Re}(A f, f), \quad f \in \operatorname{dom} A .
$$

If in addition $A$ has no sectorial extensions $(\Longleftrightarrow \rho(A) \neq \emptyset)$ it is called a $m$-sectorial operator and is put in the class $S_{\mathfrak{H}}(\varphi)$.

Further by $S_{\mathfrak{H}}(\pi / 2)$ we denote the class of m-accretive operators in $\mathfrak{H}$, i.e. $A \in S_{\mathfrak{H}}(\pi / 2)$ if $\operatorname{Re}(A f, f) \geq 0$ for all $f \in \operatorname{dom} A$ and $\rho(A) \neq \emptyset$.

Finally, $S_{\mathfrak{H}}(0)$ stands for the set of all nonnegative selfadjoint linear operators in $\mathfrak{H}$.

Following [27] an operator $A$ with $\overline{\operatorname{dom}(A)}=\mathfrak{H}$ is called regularly dissipative if $-A$ is $m$-sectorial.

Let $A$ be a closed sectorial closed operator in $\mathfrak{H}$. In the framework of the approach accepted in this paper with each $A$ it is connected a linear transformation

$$
T_{1}=X(A):=-I+2(I+A)^{-1},
$$

being a contraction with a nondense in $\mathfrak{H}$ domain of the definition $\mathfrak{H}_{1}:=\operatorname{dom}\left(T_{1}\right)=(I+$ A)dom $A$. In so doing condition (2.13) is transformed to the following one

$$
2 \cot \varphi \cdot\left|\operatorname{Im}\left(T_{1} f, f\right)\right| \leq\left(\left(I-T_{1}^{*} T_{1}\right) f, f\right)=\left\|D_{T_{1}} f\right\|^{2}, \quad f \in \operatorname{dom} T_{1} .
$$

The following definition naturally arises from what has been said.

Definition 2.9. We put an operator $T \in[\mathfrak{H}]$ in the class $C_{\mathfrak{H}}(\varphi)$ if

$$
\|T \sin \varphi \pm i \cos \varphi \cdot I\| \leq 1, \quad(\varphi \in(0, \pi / 2])
$$

and in the class $C_{\mathfrak{H}}(0)$ if $T=T^{*}$ and $\|T\| \leq 1$. 
It is clear that if $T \in C_{\mathfrak{H}}(\varphi)$, then $\sigma(T) \subset L_{\varphi}$ where

$$
L_{\varphi}:=\{z \in \mathbb{D}:|z \sin \varphi \pm i \cos \varphi| \leq 1\} .
$$

Lemma 2.10. Let $T \in[\mathfrak{H}], \quad \varphi \in(0, \pi / 2]$, Then the following properties of the operator $T$ are equivalent:

$$
\begin{array}{ll}
\text { (1) } & T \in C_{\mathfrak{H}}(\varphi) \\
\text { (2) } & 2 \cot \varphi|\operatorname{Im}(T f, f)| \leq\left\|D_{T} f\right\|^{2}, \quad f \in \mathfrak{H} ; \\
\text { (3) } & 2 \cot \varphi\left|\left(T_{I} f, g\right)\right| \leq\left\|D_{T} f\right\| \cdot\left\|D_{T} g\right\|, \quad f, g \in \mathfrak{H} .
\end{array}
$$

It follows from Lemma 2.10 that

$$
C_{\mathfrak{H}}(0)=\bigcap_{\varphi \in(0, \pi / 2)} C_{\mathfrak{H}}(\varphi) .
$$

Definition 2.11. Let $A$ be a closed sectorial operator in $\mathfrak{H}$ with vertex zero and half-angle $\varphi_{0} \in[0 ; \pi / 2)$ and let $T_{1}$ be a contraction obeying (2.15) with $\varphi=\varphi_{0}$. Denote by $\operatorname{Ext}_{A}(\varphi)$ the class of all $m$-sectorial extensions $\widetilde{A}(\in \mathcal{C}(\mathfrak{H}))$ of $A$ with vertex zero and the half-angle $\varphi \in\left[\varphi_{0} ; \pi / 2\right]$ and by $\operatorname{Ext}_{T_{1}}(\varphi)$ the class of all extensions $T \in[\mathfrak{H}]$ of $T_{1}$ obeying (2.15) with $\varphi \in\left[\varphi_{0} ; \pi / 2\right]$.

Lemma 2.12. Let $A$ be a densely defined sectorial operator in $\mathfrak{H}$ with a semiangle $\varphi$. The linear fractional transformation (2.14) establishes the bijective correspondence

$$
\widetilde{A} \rightarrow T=X(\widetilde{A})=-I+2\left(I+\widetilde{A}^{-1}, \quad T \rightarrow \widetilde{A}=X^{-1}(T)=-I+2(I+T)^{-1},\right.
$$

between the set $\operatorname{Ext}_{A}(\varphi)$ and the subset $\operatorname{Ext}_{T_{1}}^{\prime}(\varphi)=\left\{T: T \in \operatorname{Ext}_{T_{1}}(\varphi),-1 \in \rho(T)\right\}$ of $\operatorname{Ext}_{T_{1}}(\varphi)$.

If $A$ is a nondensely defined sectorial operator, then the set $\operatorname{Ext}_{A}(\varphi)$ contains $m$-sectorial linear relations too. Lemma 2.12 remains valid in this case if we replace $\operatorname{Ext}_{T_{1}}^{\prime}(\varphi)$ by $\operatorname{Ext}_{T_{1}}(\varphi)$.

\section{Some Classes of COntractive extensions of DUAl Pairs of Hermitian CONTRACTIONS}

\subsection{A parametrization of the operator loone in the special case.}

In this subsection we present an elementary result (see Lemma 3.3) on parametrization of an operator hole $L=B_{1} \cap B_{2}$ in the case of operator balls $B_{1}=B\left(Z_{1} ; R_{l}, R_{r}\right)$ and $B_{2}=B\left(Z_{2} ; R_{l}, R_{r}\right)$ with equal left radii and right radii. This lemma gives a partial solution to Problem 5 mentioned in the Introduction.

We start with the following simple lemma.

Lemma 3.1. Let $R_{1}=R_{1}^{*} \geq 0, R_{2}=R_{2}^{*} \geq 0, R_{i} \in[\mathfrak{H}], j \in\{1,2\}$, and let $A \in[\mathfrak{H}]$. Then the following conditions are equivalent:

$$
\begin{aligned}
& \text { (i) } A=R_{2} B R_{1}, \quad B \in C_{\mathfrak{H}}(\pi / 2) ; \\
& \text { (ii) } 2|(A f, g)| \leq\left(R_{1}^{2} f, f\right)+\left(R_{2}^{2} g, g\right), \quad f, g \in \mathfrak{H} .
\end{aligned}
$$

Proof. Inequality (3.2) is equivalent to the inequality

$$
|(A f, g)| \leq\left\|R_{1} f\right\| \cdot\left\|R_{2} g\right\|, \quad f, g \in \mathfrak{H} .
$$


Hence $\operatorname{ker} A \supset \operatorname{ker} R_{1}$ and $\operatorname{ker} A^{*} \supset \operatorname{ker} R_{2}$. Letting $R_{1} f=: h_{1}, R_{2} g=: h_{2}$ one rewrites (3.3) in the form

$$
\left|\left(A R_{1}^{-1} h_{1}, R_{2}^{-1} h_{2}\right)\right| \leq\left\|h_{1}\right\| \cdot\left\|h_{2}\right\|, \quad h_{1} \in \operatorname{ran} R_{1}, h_{2} \in \operatorname{ran} R_{2} .
$$

It follows that the bylinear form $\left(A R_{1}^{-1} h_{1}, R_{2}^{-1} h_{2}\right)$ may be continually extended to a bounded bylinear form $\mathfrak{t}\left(h_{1}, h_{2}\right)$ on $\mathcal{H}_{1} \times \mathcal{H}_{2}$, with $\mathcal{H}_{j}=\overline{\operatorname{ran}} R_{j}, j \in\{1,2\}$. Hence $\mathfrak{t}\left(h_{1}, h_{2}\right)=B\left(h_{1}, h_{2}\right)$, where $B \in\left[\mathcal{H}_{1}, \mathcal{H}_{2}\right]$ and $\|B\| \leq 1$. Since $\left(A R_{1}^{-1} h_{1}, R_{2}^{-1} h_{2}\right)=\left(B h_{1}, h_{2}\right), h_{j} \in \mathcal{H}_{j}, j \in\{1,2\}$, then $A R^{-1} h_{1} \in \operatorname{dom}\left(R_{2}^{-1}\right)$ and $R_{2}^{-1} A R_{1}^{-1} h_{1}=B h_{1}, \quad h_{1} \in \operatorname{dom}\left(R_{1}^{-1}\right)$. Thus, $B$ is the closure of the operator $R_{2}^{-1} A R_{1}^{-1}$. Hence $A=R_{2} B R_{1}$ and the implication (ii) $\Longrightarrow(\mathrm{i})$ is proved.

The converse implication (i) $\Longrightarrow($ ii) is clear.

The following statement easily follows from Lemma 3.1.

Lemma 3.2. Let $R_{j}=R_{j}^{*} \geq 0, R_{j} \in[\mathfrak{H}], j \in\{1,2\}$ and $A \in[\mathfrak{H}]$. Suppose additionally that ker $R_{1}=\operatorname{ker} R_{2}$ and $\mathcal{H}:=\mathfrak{H} \ominus$ ker $R_{1}$. Then $A$ admits a representation $A=R_{2} K R_{1}$ with $K \in C_{\mathcal{H}}(\varphi), \varphi \in[0, \pi / 2]$, if and only if

$$
\left.2 R e\left(\left(\sin \varphi \cdot A \pm i \cos \varphi \cdot R_{2} \cdot R_{1}\right)\right) f, g\right) \leq\left(R_{1}^{2} f, f\right)+\left(R_{2}^{2} g, g\right), \quad f, g \in \mathcal{H} .
$$

Proof. Necessity is immediately implied by Lemma 3.1 .

Sufficiency. Suppose that (3.4) is satisfied. Assume that $\varphi>0$, since the case $\varphi=0$ is trivial. Then (3.4) yields

$$
2|(A f, g)| \leq(\sin \varphi)^{-1} \cdot\left(\left\|R_{1} f\right\|^{2}+\left\|R_{2} g\right\|^{2}\right), \quad f, g \subset \mathfrak{H} .
$$

By Lemma [3.1 $A$ admits a representation $A=R_{2} K R_{1}$ with $K \in[\mathcal{H}],\|K\| \leq 1 / \sin \varphi$. Substituting this expression for $A$ in (3.4) we get the required.

The following lemma, being a partial solution to Problem 5, gives a parametrization of

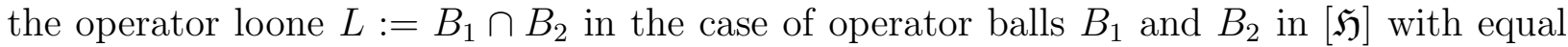
left and right radii and, in particular, it gives a criterion of nonemptyness of the loone $L$.

Lemma 3.3. Let $B_{1}=B\left(C_{1} ; R_{l}, R_{r}\right)$ and $B_{2}=B_{2}\left(C_{2} ; R_{l}, R_{r}\right)$ be two operator balls in [ $\mathfrak{H}$ ] with equal left and right radii and $\mathcal{H}_{1}:=\mathfrak{H} \ominus \operatorname{ker} R_{r}, \mathcal{H}_{2}:=\mathfrak{H} \ominus \operatorname{ker} R_{l}$. Then

(i) their intersection $L:=B_{1} \cap B_{2}$ is nonempty if and only if one of the following (equivalent) conditions is satisfied:

(a) $\left.\mid\left(C_{1}-C_{2}\right) f, g\right) \mid \leq 2^{-1}\left[\left(R_{l}^{2} f, f\right)+\left(R_{r}^{2} g, g\right)\right]$

(b) $\left.\mid\left(C_{1}-C_{2}\right) f, g\right) \mid \leq\left\|R_{l} f\right\| \cdot\left\|R_{r} g\right\|, \quad f, g \in \mathfrak{H} ;$

(c) $C_{1}-C_{2}=2 R_{l} Q R_{r} \quad$ with $\quad Q \in C(\pi / 2), Q \in\left[\mathcal{H}_{1}, \mathcal{H}_{2}\right]$;

$\left(c^{\prime}\right)$ the operator $\quad Q_{0}:=2^{-1} R_{l}^{-1}\left(C_{1}-C_{2}\right) R_{r}^{-1} \quad$ is bounded and its closure

$$
Q:=\bar{Q}_{0}\left(\in\left[\mathcal{H}_{1}, \mathcal{H}_{2}\right]\right) \text { is a contraction. }
$$

(ii) If any of the conditions (a), (b), (c) is satisfied, then the operator loone $L$ admits the following parameter representation

(iii) $L$ consists of one element, $L=\left\{2^{-1}\left(C_{1}+C_{2}\right)\right\}$, if and only if at least one of the following three conditions holds
(1) $R_{l}=0$;
(2) $\quad R_{r}=0$;
(3) $Q$ is a maximal partial isometry. 
Proof. (i), (ii). Equivalence of the conditions (a)-(c) is implied by Lemma 3.1. It remains to show, for example, that equality (3.7) is equaivalent to the condition $L \neq \varnothing$. Let $T \in L=$ $B_{1} \cap B_{2}$, that is

$$
T=C_{1}+R_{l} K_{1} R_{r}=C_{2}+R_{l} K_{2} R_{r}
$$

Then setting

$$
K:=2^{-1}\left(K_{1}+K_{2}\right)(\in C(\pi / 2)) \quad \text { and } \quad Q:=2^{-1}\left(K_{2}-K_{1}\right)(\in C(\pi / 2)),
$$

we deduce

$$
T=2^{-1}\left(C_{1}+C_{2}\right)+R_{l} K R_{r}, \quad \text { where } \quad K \pm Q \in C(\pi / 2) .
$$

Thus, conditions (3.7) and (3.8) are satisfied.

Conversely, suppose that (3.7) is valid. Then setting $K_{1}:=-Q, K_{2}:=+Q$ and $T:=$ $C_{1}+R_{l} K_{1} R_{r}$ we get

$$
T=C_{1}+R_{l} K_{1} R_{r}=C_{2}+R_{l} K_{2} R_{r}
$$

Hence $T \in L=B_{1} \cap B_{2}$.

(iii) Let $T \in L$ and $T \neq 2^{-1}\left(C_{1}+C_{2}\right)$. Then according to $(3.8) R_{l} \neq 0, R_{r} \neq 0$ and there exists $K \in C(\pi / 2) \backslash\{0\}$ such that $K_{ \pm}:=Q \pm K \in C(\pi / 2)$. Hence $Q=\left(K_{+}+K_{-}\right) / 2 \neq$ $K_{+} \neq K_{-}$is not an extreme point of the unit ball in $\left[\mathfrak{H}_{1}, \mathfrak{H}_{2}\right]$. By Proposition [2.7] $Q$ is not a maximal isometry.

Conversely, suppose that $R_{l} \neq 0, R_{r} \neq 0$ and $Q$ is not a maximal isometry. By Proposition $2.7 Q=\left(K_{+}+K_{-}\right) / 2$ where $K_{ \pm} \in\left[\mathcal{H}_{1}, \mathcal{H}_{2}\right], K_{ \pm} \in C(\pi / 2)$ and $K_{+} \neq K_{-}$. Setting $K_{1}:=\left(K_{+}-K_{-}\right) / 2$ and $K_{2}:=-K_{1}$ we easily get that $K_{j} \pm Q \in C(\pi / 2), j \in\{1,2\}$. Hence by (3.8) $T_{K_{j}}:=2^{-1}\left(C_{1}+C_{2}\right)+R_{l} K_{j} R_{r} \in L, j \in\{1,2\}$. Since $T_{K_{j}} \neq 2^{-1}\left(C_{1}+C_{2}\right)$, we get the required.

3.2. A description of the class of $C_{\mathfrak{H}}(\varphi)$-extensions of a dual pair of symmetric contractions. In this subsection we present a solution to the Problem $2 \mathrm{C}$ with $\varphi_{0}=0$.

More precisely, let $\left\{T_{1}, T_{2}\right\}$ be a dual pair of symmetric contractions in $\mathfrak{H}=\mathfrak{H}_{1} \oplus \mathfrak{H}_{2}=\mathfrak{H}_{1}^{\prime} \oplus$ $\mathfrak{H}_{2}^{\prime}$. Due to (2.11) the operators $T_{1}$ and $T_{2}$ admit the following block-matrix representations

$$
T_{1}=\left(\begin{array}{c}
T_{11} \\
T_{21}
\end{array}\right)=\left(\begin{array}{c}
T_{11} \\
V D_{T_{11}}
\end{array}\right), \quad T_{2}=\left(\begin{array}{c}
T_{11} \\
T_{12}^{*}
\end{array}\right)=\left(\begin{array}{c}
T_{11} \\
U^{*} D_{T_{11}}
\end{array}\right),
$$

since $T_{11}=T_{11}^{*}$. In particular, in this case $\mathfrak{H}_{1}=\mathfrak{H}_{1}^{\prime}$ and $\mathfrak{H}_{2}=\mathfrak{H}_{2}^{\prime}$.

Let

$$
\operatorname{Ext}_{\left\{T_{1}, T_{2}\right\}}(\varphi):=\operatorname{Ext}_{\left\{T_{1}, T_{2}\right\}} \cap C_{\mathfrak{H}}(\varphi), \quad \varphi \in[0, \pi / 2],
$$

stand for the set of $C_{\mathfrak{H}}(\varphi)$-extensions of the dual pair of symmetric contractions $\left\{T_{1}, T_{2}\right\}$.

By Theorem 2.4 $\operatorname{Ext}_{\left\{T_{1}, T_{2}\right\}}(\pi / 2) \neq \emptyset$, that is there always exists an extension $T \in C_{\mathfrak{H}}(\pi / 2)$ of the dual pair $\left\{T_{1}, T_{2}\right\}$. It turns out that it is not the case for the classes $C_{\mathfrak{H}}(\varphi)$ and $C_{\mathfrak{H}}(\varphi ; \varkappa)$ with $\varphi<\pi / 2$. The solvability of both problems depends on the properties of the operator

$$
Q_{0}:=D_{V^{*}}^{-1}(I-V U) D_{U}^{-1} .
$$

Moreover, we show that if the operator $Q_{0}$ is unbounded the Problem 2C has a solution only with $\varphi_{1}=\pi / 2$, that is, $\operatorname{Ext}_{\left\{T_{1}, T_{2}\right\}}(\varphi) \neq \emptyset$ iff $\varphi=\varphi_{1}=\pi / 2$. 
Theorem 3.4. Let $\left\{T_{1}, T_{2}\right\}$ be a dual pair of symmetric contractions in $\mathfrak{H}=\mathfrak{H}_{1} \oplus \mathfrak{H}_{2}$ of the form (3.9),$\varphi \in(0, \pi / 2]$ and let $\mathcal{H}_{1}:=\overline{\operatorname{ran}}\left(D_{U}\right), \mathcal{H}_{2}:=\overline{\operatorname{ran}}\left(D_{V^{*}}\right)$. Then:

(i) the set $\operatorname{Ext}_{\left\{T_{1}, T_{2}\right\}}(\varphi)$ is nonempty if and only if $\varphi \in\left[\varphi_{1}, \pi / 2\right]$, where

$$
\varphi_{1}:=\arccos \left(\left\|D_{V^{*}}^{-1}(I-V U) D_{U}^{-1}\right\|^{-1}\right) ;
$$

(ii) for any $\varphi \in\left[\varphi_{1}, \pi / 2\right]$ the following equivalence holds:

$$
\begin{aligned}
T_{K}=\left(\begin{array}{ll}
T_{11} & T_{12} \\
T_{21} & T_{22}
\end{array}\right) \in \operatorname{Ext}_{\left\{T_{1}, T_{2}\right\}}(\varphi) \Longleftrightarrow \\
T_{22}=-V T_{11} U+D_{V^{*}} K D_{U}, \quad K \cdot \sin \varphi \pm i Q \cos \varphi \in C(\pi / 2),
\end{aligned}
$$

where $Q:=\bar{Q}_{0}\left(\in\left[\mathcal{H}_{1}, \mathcal{H}_{2}\right]\right)$ is the closure of the operator $Q_{0}$ of the form (3.11) and $K \in$ $\left[\mathcal{H}_{1}, \mathcal{H}_{2}\right]$

(iii) the set $\operatorname{Ext}\left\{T_{1}, T_{2}\right\}(\varphi)$ consists of one element if at least one of the following three conditions is satisfied:
(a) $D_{V^{*}}=0$;
(b) $D_{U}=0$;
(c) $Q \cos \varphi$ is a maximal partial isometry.

Proof. (i) Let $T=\left(T_{i j}\right)_{i, j=1}^{2}$ be a contractive extension of the dual pair $\left\{T_{1}, T_{2}\right\}$. Suppose for the begining that $\varphi>0$. In this case the inclusion $T \in C_{\mathfrak{H}}(\varphi)$ means that

$$
T_{ \pm}:=T \sin \varphi \pm i \cos \varphi \cdot I \in C_{\mathfrak{N}}(\pi / 2),
$$

that is,

$$
T_{ \pm}:=\left(\begin{array}{ll}
B_{11}^{ \pm} & B_{12} \\
B_{21} & B_{2}^{ \pm}
\end{array}\right):=\left(\begin{array}{cc}
\sin \varphi \cdot T_{11} \pm i \cos \varphi \cdot I & T_{21} \sin \varphi \\
T_{21} \sin \varphi & \sin \varphi \cdot T_{22} \pm i \cos \varphi \cdot I
\end{array}\right) \in C_{\mathfrak{H}}(\pi / 2) .
$$

It is easily seen that

$$
\begin{aligned}
& D_{B_{11}^{+}}^{2}=I-\left(\sin \varphi \cdot T_{11}-i \cos \varphi \cdot I\right)\left(\sin \varphi \cdot T_{11}+i \cos \varphi \cdot I\right)=\sin ^{2} \varphi \cdot D_{T_{11}}^{2}, \\
& D_{B_{11}^{-}}^{2}=I-\left(\sin \varphi \cdot T_{11}+i \cos \varphi \cdot I\right)\left(\sin \varphi \cdot T_{11}-i \cos \varphi \cdot I\right)=\sin ^{2} \varphi \cdot D_{T_{11}}^{2} .
\end{aligned}
$$

Therefore $D_{B_{11}^{+}}=D_{B_{11}^{-}}=\sin \varphi \cdot D_{T_{11}}$ and consequently

$$
\begin{aligned}
& B_{12}=\sin \varphi T_{12}=\sin \varphi \cdot D_{T_{11}} U=D_{B_{11}^{ \pm}} U, \\
& B_{21}=\sin \varphi T_{21}=\sin \varphi \cdot V D_{T_{11}}=V D_{B_{11}^{ \pm}} .
\end{aligned}
$$

Thus the contractions $T_{ \pm}$have the form

$$
T_{ \pm}=\left(\begin{array}{cc}
B_{11}^{ \pm} & B_{12} \\
B_{21} & B_{22}^{ \pm}
\end{array}\right)=\left(\begin{array}{cc}
B_{11}^{ \pm} & D_{B_{11}^{ \pm}} U \\
V D_{B_{11}^{ \pm}} & B_{22}^{ \pm}
\end{array}\right)
$$

with $B_{11}^{ \pm}=T_{11} \sin \varphi \pm i \cos \varphi \cdot I$ and $B_{22}^{ \pm}=T_{22} \sin \varphi \pm i \cos \varphi \cdot I$. According to Theorem 2.4 the equivalences

$$
T_{ \pm} \in C(\pi / 2) \Longleftrightarrow B_{22}^{+}=T_{22} \sin \varphi \pm i \cos \varphi \cdot I=C_{ \pm}+D_{V^{*}} K_{ \pm} D_{U}
$$

hold true with some contractions $K_{ \pm}$and operators $C_{ \pm}$defined by

$$
C_{ \pm}:=-V\left(B_{11}^{ \pm}\right)^{*} U=-V\left(\sin \varphi \cdot T_{11}^{*} \mp i \cos \varphi\right) U \text {. }
$$

Setting

$$
C_{1}:=C_{+}-i \cos \varphi \cdot I, \quad C_{2}:=C_{-}+i \cos \varphi \cdot I
$$


we rewrite equivalences $(3.16)$ in the form

$$
T \in C_{\mathfrak{H}}(\varphi) \Longleftrightarrow T_{22} \sin \varphi=C_{1}+D_{V^{*}} K_{+} D_{U}=C_{2}+D_{V^{*}} K_{-} D_{U}, \quad K_{ \pm} \in C(\pi / 2) .
$$

Thus, $T \in C_{\mathfrak{H}}(\varphi)$ if and only if the operator $\sin \varphi \cdot T_{22}$ belongs to the intersection of the operator balls $B_{1}:=B\left(C_{1} ; D_{V^{*}} ; D_{U}\right)$ and $B_{2}:=B\left(C_{2} ; D_{V^{*}}, D_{U}\right)$, that is, $\sin \varphi \cdot T_{22} \in L:=$ $B_{1} \cap B_{2}$. By Lemma 3.3 with account of (3.17) and (3.18) the condition $L=B_{1} \cap B_{2} \neq \varnothing$ amounts to saying that the operator

$$
C_{0}:=2^{-1}\left(C_{1}-C_{2}\right)=-i \cos \varphi(I-V U)
$$

admits the representation $C_{0}=D_{V^{*}} K D_{U}$ with $K \in C(\pi / 2)$ or, what is the same, the operator $Q_{0} \cdot \cos \varphi$ is contractive where the operator $Q_{0}$ is of the form (3.11). This proves the first assertion.

(ii) Suppose that condition (3.12) is satisfied. To obtain a parametrization of the hole $L$ we note that by (3.17) and (3.18)

$$
2^{-1}\left(C_{1}+C_{2}\right)=-\sin \varphi \cdot V T_{11}^{*} U
$$

Now Lemma 3.3 yields the equivalence

$$
T \in L\left(=B_{1} \cap B_{2}\right) \Longleftrightarrow \sin \varphi \cdot T_{22}=-\sin \varphi \cdot V T_{11}^{*} U+D_{V^{*}} \widetilde{K} D_{U},
$$

where $\widetilde{K} \pm i Q \cos \varphi \in C(\pi / 2)$. Setting in (3.21) $K:=\widetilde{K} / \sin \varphi$ we arrive at (3.13).

It remains to consider the case $T \in \operatorname{Ext}_{\left\{T_{1}, T_{2}\right\}}(0)$. This inclusion means that $T$ is a selfadjoint contraction in $\mathfrak{H}$, that is $T_{21}=T_{12}^{*}$ and $U=V^{*}$. Hence $Q_{0}=I$ and $\mathcal{H}_{1}=\mathcal{H}_{2}=: \mathcal{H}$. Threfore equivalence (3.13) with $\varphi \in(0, \pi / 2)$ takes the form

$$
T_{K} \in C_{\mathfrak{H}}(\varphi) \Longleftrightarrow K \cdot \sin \varphi \pm i \cos \varphi \cdot I \in C_{\mathcal{H}}(\pi / 2) .
$$

The desired equivalence

$$
T_{K} \in C_{\mathfrak{H}}(0) \Longleftrightarrow K=K^{*} \in C_{\mathcal{H}}(\pi / 2)
$$

is implied now by (2.18).

(iii) This assertion is immediately implied by the statement (iii) of Lemma 3.3 .

Remark 3.5. Comparison of condition (3.12) with the obvious criterion $U=V^{*}$ for the existence of $T=T^{*} \in \operatorname{Ext}_{\left\{T_{1}, T_{1}\right\}}(\pi / 2)$ yields a curious fact:

$$
U, V \in C_{\mathfrak{H}}(\pi / 2), \quad|((I-V U) f, g)| \leq\left\|D_{U} f\right\| \cdot\left\|D_{V^{*}} g\right\| \Longleftrightarrow U=V^{*} .
$$

I don't know the direct proof if this equivalence.

3.3. Extreme points of the set $\operatorname{Ext}_{\left\{T_{1}, T_{2}\right\}}(\varphi)$. Denote by $\operatorname{Ext}_{\left\{T_{1}, T_{2}\right\}}^{e}(\varphi)$ the set of extreme points of the closed convex set $\operatorname{Ext}_{\left\{T_{1}, T_{2}\right\}}(\varphi)$. Theorem 3.4 makes it possible to describe a part of the set $\operatorname{Ext}_{\left\{T_{1}, T_{2}\right\}}^{e}(\varphi)$. To this end for any operator $Q\left(\in\left[\mathcal{H}_{1}, \mathcal{H}_{2}\right]\right)$ we introduce the operator loones

$$
L(Q ; \varphi):=\left\{K \in\left[\mathcal{H}_{1}, \mathcal{H}_{2}\right]: K \sin \varphi \pm i Q \cos \varphi \in C(\pi / 2)\right\}, \quad \varphi \in(0, \pi / 2),
$$

and denote by $L^{e}(Q ; \varphi)$ the set of its extreme points.

Proposition 3.6. Let $Q \in\left[\mathcal{H}_{1}, \mathcal{H}_{2}\right], \varphi_{1}:=\arccos \left(\|Q\|^{-1}\right)>0$ and $\varphi \in\left[\varphi_{1}, \pi / 2\right]$. Then

(i) the following equivalence holds

$$
K \in L(Q ; \varphi) \Longleftrightarrow \sin 2 \varphi \cdot\left(K^{*} Q\right)_{I}=D_{K, Q} C D_{K, Q}, \quad C=C^{*} \in C_{\mathcal{H}_{1}}(0),
$$


where $\left(K^{*} Q\right)_{I}:=(2 i)^{-1}\left(K^{*} Q-Q^{*} K\right)$ and

$$
D_{K, Q}:=\left(I-K^{*} K \sin ^{2} \varphi-Q^{*} Q \cos ^{2} \varphi\right)^{1 / 2} \geq 0 .
$$

(ii) If additionally $\operatorname{ran} D_{K, Q}$ is closed, that is $\operatorname{ran} D_{K, Q}=\overline{\operatorname{ran}} D_{K, Q}$, then the following implication holds

$$
\sigma(C) \subset\{ \pm 1\} \Longrightarrow K \in L^{e}(Q ; \varphi) .
$$

Proof. (i) By definition $K \in L(Q ; \varphi)$ iff

$$
\left(I-K^{*} \sin \varphi \mp i Q^{*} \cos \varphi\right)(K \sin \varphi \pm i Q \cos \varphi) \geq 0 .
$$

With account of definition (3.27) this inequality may be rewritten as

$$
\pm \sin 2 \varphi\left(K^{*} Q\right)_{I} \leq D_{K, Q}^{2}
$$

By Lemma 3.1 this inequality is equivalent to representation (3.26) with some selfadjoint contraction $C$.

(ii) The proof of this statement is similar to that of Proposition 3.18 from [30]. Suppose the contrary, that is $K \notin L^{e}(Q ; \varphi)$. Then $2 K=K_{1}+K_{2}$ where $K_{j} \in L(Q ; \varphi), j \in\{1,2\}$ and $K_{1} \neq K$. For any $f \in \operatorname{ker} D_{K, Q}$ we have

$$
\begin{aligned}
& 4\|f\|^{2}=\sin ^{2} \varphi \cdot\|2 K\|^{2}+4 \cos ^{2} \varphi \cdot\|Q f\|^{2}=\sin ^{2} \varphi \cdot\left\|K_{1} f+K_{2} f\right\|^{2}+4 \cos ^{2} \varphi \cdot\|Q f\|^{2} \\
& \leq 2\left(\sin ^{2} \varphi \cdot\left\|K_{1} f\right\|^{2}+\cos ^{2} \varphi \cdot\|Q f\|^{2}\right)+2\left(\sin ^{2} \varphi \cdot\left\|K_{2} f\right\|^{2}+\cos ^{2} \varphi \cdot\|Q f\|^{2}\right) \leq 4\|f\|^{2} .
\end{aligned}
$$

Hence

$$
\sin ^{2} \varphi \cdot\left\|K_{j} f\right\|^{2}+\cos ^{2} \varphi \cdot\|Q f\|^{2}=\|f\|^{2}, \quad j \in\{1,2\} .
$$

Thus, $\left\|K_{1} f\right\|=\left\|K_{2} f\right\|=\|K f\|$ and $\left\|K_{1} f+K_{2} f\right\|=\left\|K_{1} f\right\|+\left\|K_{2} f\right\|$. In view of strict convexity of the unit ball in $\mathfrak{H}$ we get

$$
K_{1} f=K_{2} f=K f, \quad f \in \operatorname{ker} D_{K, Q} .
$$

Further, setting $K_{ \pm}:=K \sin \varphi \pm i Q \cos \varphi$ and using representation (3.26) we obtain

$$
D_{K_{ \pm}}^{2}=D_{K, Q}^{2} \pm \sin 2 \varphi \cdot\left(K^{*} Q\right)_{I}=D_{K, Q}(I \pm C) D_{K, Q} .
$$

Suppose that $f \in\left(\operatorname{ker} D_{K, Q}\right)^{\perp}$ and $D_{K, Q} f \in \operatorname{ker}(I+C)$. Then, it follows from (3.30) that $D_{K_{+}}^{2} f=0$, that is $\left\|K_{+} f\right\|=\|f\|$. Setting

$$
K_{J+}:=K_{j} \sin \varphi+i Q \cos \varphi(\in C(\pi / 2)), \quad j \in\{1,2\},
$$

and noting that $2 K_{+}=K_{1+}+K_{2+}$ we easily get

$$
2\|f\|=2\left\|K_{+} f\right\|=\left\|\left(K_{1+}+K_{2+}\right) f\right\| \leq 2\|f\| .
$$

Hence $\left\|K_{1+} f\right\|=\left\|K_{2+} f\right\|=\left\|K_{+} f\right\|=\|f\|$ and

$$
\left\|K_{1+} f+K_{2+} f\right\|=\left\|K_{1+} f\right\|+\left\|K_{2+} f\right\| .
$$

In view of strict convexity of the unit ball in $\mathfrak{H}$ we get $K_{1+} f=K_{2+} f=K_{+} f$, that is $K_{1} f=K_{2} f=K f$ for any $f$ obeying $D_{K, Q} f \in \operatorname{ker}(I+Q)$. Similarly we obtain that $K_{1} f=K_{2} f=K f$ for any $f$ such that $D_{K, Q} f \in \operatorname{ker}(I-Q)$. Taking into account the hypothesis of proposition we get

$$
K_{1} f=K_{2} f=K f, \quad f \in \operatorname{ran} D_{K, Q}=k e r(I+C) \oplus \operatorname{ker}(I-C) .
$$

Combining (3.29) with (3.31) we get $K=K_{1}=K_{2}$. This contradicts the assumption that $K_{1} \neq K$. 
Remark 3.7. (a) Closability of the linear manifolds ran $D_{K, Q}$ in Porposition 3.6 may be replaced by $\overline{\operatorname{ran} D_{K, Q} \cap \mathcal{H}_{ \pm}}=\mathcal{H}_{ \pm}$where $\mathcal{H}_{ \pm}:=\operatorname{ker}(I \pm C)$, which are, for example, valid if either $\operatorname{dim} \mathcal{H}_{+}<\infty$ or $\operatorname{dim} \mathcal{H}_{-}<\infty$.

(b) Note that $\operatorname{ran} D_{K, Q}$ is closed if both $K$ and $Q$ are compact operators, $K, Q \in \mathfrak{S}_{\infty}$.

Next we clarify and complement Proposition 3.6] in the case $\mathcal{H}_{1}=\mathcal{H}_{2}=\mathcal{H}$ and $Q=I_{\mathcal{H}}$. Now $L(Q ; \varphi)=L\left(I_{\mathcal{H}} ; \varphi\right)=C_{\mathcal{H}}(\varphi)$. Denote by $C_{\mathcal{H}}^{e}(\varphi):=L^{e}\left(I_{\mathcal{H}} ; \varphi\right)$ the set of extreme points of the set $C_{\mathcal{H}}(\varphi)$ and by

$$
\partial L_{\varphi}:=\partial L_{\varphi}^{+} \cup \partial L_{\varphi}^{-} \quad \text { where } \quad \partial L_{\varphi}^{ \pm}:=\{z \in \mathbb{D}:|z \sin \varphi \pm i \cos \varphi|=1\},
$$

the (topological) boundary of the hole (2.17). Note that $\partial L_{\varphi}$ is at the same time the set of extreme points of the hole (2.17).

Proposition 3.8. Let $\varphi \in(0, \pi / 2)$ and $K \in C_{\mathcal{H}}(\varphi)$. Then

(i) there exists a contraction $C=C^{*}$ such that

$$
2 K_{I}=\tan \varphi \cdot D_{K} C D_{K}, \quad C \in C_{\mathcal{H}}(0) .
$$

Conversly, if $K \in C_{\mathcal{H}}(\pi / 2)$ and (3.33) holds then $K \in C_{\mathcal{H}}(\varphi)$;

(ii) the following implication holds

$$
\sigma(C) \subset\{ \pm 1\} \quad \text { and } \overline{\operatorname{ran} D_{K, Q} \cap \mathcal{H}_{ \pm}}=\mathcal{H}_{ \pm}:=\operatorname{ker}(I \pm C) \Longrightarrow K \in C_{\mathcal{H}}^{e}(\varphi)
$$

(iii) if $K$ is a normal operator, $K K^{*}=K^{*} K$, and $\sigma(K) \subset \partial L_{\varphi}$ then $K \in C_{\mathcal{H}}^{e}(\varphi)$;

(iv) if $K \in C_{\mathcal{H}}(\varphi), \sigma(K) \subset \partial L_{\varphi}$ and the spectrum $\sigma(K)$ is purely point, then $K$ is normal, hence $K \in C_{\mathcal{H}}^{e}(\varphi)$.

(v) the $\operatorname{set} C_{\mathcal{H}}^{e}(\varphi)$ contains continuum (nonnormal) operators $K$ with $\sigma(K)=0$.

Proof. (i) If $Q=I_{\mathcal{H}}$ then $D_{K, Q}=D_{K, I}=\sin \varphi \cdot D_{K}$ and the statement is implied by Proposition 3.6 (i).

(ii) This statement is implied by Proposition 3.6 (ii).

(iii) Assume for brevity that $\pm 1 \notin \sigma_{p}(K)$. Then starting with (3.33) and applying Spectral theorem we get

$$
\begin{array}{r}
C=\cot \varphi \cdot D_{K}^{-1}\left(2 K_{I}\right) D_{K}^{-1}=\cot \varphi \cdot \int_{\partial L_{\varphi}} \frac{\lambda-\bar{\lambda}}{i \sqrt{1-|\lambda|^{2}}} d E_{K}(\lambda) \\
=\cot \varphi \cdot \int_{\partial L_{\varphi}^{+}} \frac{\lambda-\bar{\lambda}}{i \sqrt{1-|\lambda|^{2}}} d E_{K}(\lambda)+\cot \varphi \cdot \int_{\partial L_{\varphi}^{-}} \frac{\lambda-\bar{\lambda}}{i \sqrt{1-|\lambda|^{2}}} d E_{K}(\lambda) \\
=\int_{\partial L_{\varphi}^{+}} d E_{K}(\lambda)-\int_{\partial L_{\varphi}^{-}} d E_{K}(\lambda)=: P_{+}-P_{-} .
\end{array}
$$

Here $E_{K}(\cdot)$ is the spectral measure of $K$, and $P_{ \pm}$are the corresponding spectral projections. Since $P_{+}+P_{-}=I$, we have $\sigma(C) \subset\{ \pm 1\}$. Moreover, $P_{ \pm} \operatorname{ran} D_{K}$ is dense in $\mathcal{H}_{ \pm}:=P_{ \pm} \mathcal{H}$. Hence by statement (ii) $K \in C_{\mathcal{H}}^{e}(\varphi)$.

(iv) Let us set $K_{ \pm}:=K \sin \varphi \pm i \cos \varphi$. If $\lambda_{j} \in \partial L_{\varphi} \cap \sigma_{p}(K)$ and $\mathcal{H}_{j}:=\operatorname{ker}\left(K-\lambda_{j}\right)(\neq \emptyset)$, then either $\mu_{j}^{+}:=\lambda_{j} \sin \varphi+i \cos \varphi \in \sigma_{p}\left(K_{+}\right) \cap \mathbb{T}$ or $\mu_{j}^{-}:=\lambda_{j} \sin \varphi-i \cos \varphi \in \sigma_{p}\left(K_{-}\right) \cap \mathbb{T}$ where $\mathbb{T}:=\{z \in \mathbb{C}:|z|=1\}$. Thus, the subspace $\mathcal{H}_{j}$ reduces the operator $K$ for any $j \in \mathbb{Z}_{+}$since either $\mathcal{H}_{j}=\operatorname{ker}\left(K_{+}-\mu_{j}^{+}\right)$or $\mathcal{H}_{j}=\operatorname{ker}\left(K_{-}-\mu_{j}^{-}\right)$and both $K_{+}$and $K_{-}$ are contractions. Since the spectrum $\sigma(K)$ is purely point, then $K=\bigoplus_{j=1}^{\infty} \lambda_{j} I_{\mathcal{H}_{j}}$ and $K$ is normal. 
(v) First we consider the case $\mathcal{H}=\mathbb{C}^{2}$. We let

$$
K(\theta):=e^{i \theta}\left(\begin{array}{cc}
0 & \sin \varphi \\
0 & 0
\end{array}\right), \quad \theta \in[0,2 \pi] .
$$

Then

$$
D_{K_{(\theta)}}=\left(\begin{array}{cc}
1 & 0 \\
0 & \cos \varphi
\end{array}\right), \quad K(\theta)_{I}=i \sin \varphi\left(\begin{array}{cc}
0 & -e^{i \theta} \\
e^{-1 \theta} & 0
\end{array}\right), \quad C(\theta)=i\left(\begin{array}{cc}
0 & -e^{-i \theta} \\
e^{-i \theta} & 0
\end{array}\right) .
$$

Hence $\sigma(C(\theta))=\{ \pm 1\}$ and by statement (ii) $K(\theta) \in C_{\mathcal{H}}^{e}(\varphi)$.

Remark 3.9. (i) Another proof of statement (iii) is contained in [30]. The proof of statement (iv) is borrowed from [30] and it is presented for the sake if completeness.

(ii) Note that while a complete description of the set $C_{\mathcal{H}}^{e}(\varphi)$ is unknown, it essentially differs from that of the sets $C_{\mathcal{H}}^{e}(0)$ and $C_{\mathcal{H}}^{e}(\pi / 2)$ even in the case $\operatorname{dim} \mathcal{H}<\infty$. Indeed, if $\operatorname{dim} \mathcal{H}<\infty$ then by Proposition 2.7 both $C_{\mathcal{H}}^{e}(\pi / 2)$ and $C_{\mathcal{H}}^{e}(0)$ consist of normal matrices with "boundary spectrum", that is, $C^{e}(\pi / 2)$ (resp. $\left.C_{\mathcal{H}}^{e}(0)\right)$ is the set of unitary (resp. unitary selfadjoint) matrices.

On the other hand, the sets $C_{\mathfrak{H}}(\varphi), \varphi \in(0, \pi)$ may be considered as "interpolation sets" between $C_{\mathfrak{H}}(0)$ and $C_{\mathfrak{H}}(\pi / 2)$. This observation makes natural the following hypothesis: for any $\varphi \in(0, \pi)$ the set $C_{\mathfrak{H}}(\varphi)$ consists of normal matrices with "boundary spectrum". However Proposition 3.8 shows that this hypothesis is false to be true, since the set $C_{\mathfrak{H}}^{e}(\varphi)$ contains continuum nonnormal matrices in addition to the set of normal matrices with spectrum lying on $\partial L_{\varphi}$.

Combining Theorem 3.4 with Proposition 3.6 we arrive at the following result.

Corollary 3.10. Suppose that conditions of Theorem 3.4 are satisfied, $Q_{0}$ and $\varphi_{1}$ are defined by (3.11) and (3.12) respectively, and $T_{K} \in \operatorname{Ext}_{\left\{T_{1}, T_{2}\right\}}(\varphi)$. Then

(i) for any $\varphi \in\left[\varphi_{1}, \pi / 2\right]$ the following equivalence holds

$$
T_{K} \in \operatorname{Ext}_{\left\{T_{1}, T_{2}\right\}}^{e}(\varphi) \Longleftrightarrow K \in L^{e}(Q ; \varphi) ;
$$

(ii) there exists a selfadjoint contraction $C \in C_{\mathcal{H}_{1}}(0)$ such that

$$
\sin 2 \varphi \cdot\left(K^{*} Q\right)_{I}=D_{K, Q} C D_{K, Q}
$$

(iii) the following implication holds

$$
\sigma(C)=\{ \pm 1\} \quad \text { and } \quad \operatorname{ran} D_{K, Q}=\overline{\operatorname{ran}} D_{K, Q} \Longrightarrow T_{K} \in \operatorname{Ext}_{\left\{T_{1}, T_{2}\right\}}^{e}(\varphi)
$$

(iv) $T_{K} \in \operatorname{Ext}_{\left\{T_{1}, T_{2}\right\}}^{e}(\varphi)$ if at least one of the following identities holds

$$
D_{K, Q}^{2} \pm \sin 2 \varphi \cdot\left(K^{*} Q\right)_{I}=0, \quad D_{K^{*}, Q^{*}}^{2} \pm \sin 2 \varphi\left(K^{*} Q\right)_{I}=0 .
$$

3.4. Proper $C_{\mathfrak{H}}(\varphi)$-extensions of symmetric contractions. Here we apply Theorem 3.4 to the case of a dual pair $\left\{T_{1}, T_{1}\right\}$.

Let $T_{1} \in\left[\mathfrak{H}_{1}, \mathfrak{H}\right]$ be a nondensely defined symmetric contraction in $\mathfrak{H}=\mathfrak{H}_{1} \oplus \mathfrak{H}_{2}$. As usual $\operatorname{Ext}_{T_{1}}$ stands for the set of all proper extensions of $T_{1}$, that is $T \in \operatorname{Ext}_{T_{1}}$ iff $T \supset T_{1}$ and $T^{*} \supset T_{1}$. Denote by

$$
\operatorname{Extp}_{T_{1}}(\varphi):=\operatorname{Ext}_{T_{1}} \cap C_{\mathfrak{H}}(\varphi), \quad \varphi \in[0, \pi / 2],
$$

the set of all proper $C_{\mathfrak{H}}(\varphi)$-extensions of the symmetric contraction $T_{1}$. 
By Definition 2.1 Ext $\operatorname{sT}_{\left.1, T_{1}\right\}}=\operatorname{Ext}_{T_{1}}$. Moreover, it follows from (3.10) and (3.35) that $\operatorname{Ext}_{\left\{T_{1}, T_{1}\right\}}(\varphi)=\operatorname{Extp}_{T_{1}}(\varphi)$.

Corollary 3.11. Let $T_{1} \in\left[\mathfrak{H}_{1}, \mathfrak{H}\right]$ be a nondensely defined symmetric contraction in $\mathfrak{H}=$ $\mathfrak{H}_{1} \oplus \mathfrak{H}_{2}$. Then $\operatorname{Extp}_{T_{1}}(\varphi)=\operatorname{Ext}_{\left\{T_{1}, T_{1}\right\}}(\varphi) \neq \varnothing$ for any $\varphi \in[0, \pi / 2]$. Moreover, the following equivalence holds

$$
T:=T_{K} \in \operatorname{Extp}_{T_{1}}(\varphi) \Longleftrightarrow T_{22}=-U^{*} T_{11} U+D_{U} K D_{U}, \quad K \in C_{\mathcal{H}}(\varphi),
$$

where $\mathcal{H}:=\mathfrak{H}_{2} \ominus \operatorname{ker} D_{U}$.

Proof. According to (3.9) $T_{1}=T_{2}$ if and only if $T_{12}=T_{21}^{*}$, that is iff $V^{*}=U$. Therefore the operator $Q_{0}$ defined by (3.11) takes the form $Q_{0}=I_{\mathcal{H}}$, where $I_{\mathcal{H}}$ is the identical operator in $\mathcal{H}$. Thus $\varphi_{1}=\arccos \left(\left\|Q_{0}\right\|^{-1}\right)=\arccos 1=0$ and $\operatorname{Extp}_{T_{1}}(\varphi) \neq \varnothing$ for any $\varphi \in[0, \pi / 2]$. Moreover, now equivalence (3.13) takes the form (3.36).

Remark 3.12. In the case $T_{1}=T_{2}$ both left and right radii of the balls $B_{1}$ and $B_{2}$ are equal: $R_{l}=D_{V^{*}}=D_{U}=R_{r}$.

According to (3.13) the set Ext $T_{1}(0)$ of selfadjoint contractive extensions of $T_{1}$ forms an operator segment ("the self-adjoint part" of the operator ball $B\left(-U^{*} T_{11} U ; D_{U}, D_{U}\right)$ ) which is parametrized by the operator segment $\left\{K \in[\mathcal{H}]:-I_{\mathcal{H}} \leq K \leq I_{\mathcal{H}}\right\}$.

Consider the extremal selfadjoint contractive extensions $T_{m}:=T_{\min }$ and $T_{M}:=T_{\max }$ of the operator $T_{1}$. It is clear that $T_{m}:=T_{-I}$ and $T_{M}:=T_{I}$ are the extreme points of the segment $\operatorname{Extp}_{T_{1}}(0)$, corresponding to the operators $K=-I_{\mathcal{H}}$ and $K=I_{\mathcal{H}}$ respectively. Their block-matrix representations are of the form

$$
T_{m}=\left(\begin{array}{cc}
T_{11} & D_{T_{11}} U \\
U^{*} D_{T_{11}} & -I+U^{*}\left(I-T_{11}\right) U
\end{array}\right), \quad T_{M}=\left(\begin{array}{cc}
T_{11} & T_{D_{11}} U \\
U^{*} D_{T_{11}} & I-U^{*}\left(I+T_{11}\right) U
\end{array}\right)
$$

Using representations (3.37) we rewrite description (3.36) as

$$
T_{K} \in \operatorname{Extp}_{T_{1}}(\varphi) \Longleftrightarrow 2 T_{K}=\left(T_{M}+T_{m}\right)+\left(T_{M}-T_{m}\right)^{1 / 2} K\left(T_{M}-T_{m}\right)^{1 / 2}, \quad K \in C_{\mathcal{H}}(\varphi) \text {. }
$$

Note that this description of the class $\operatorname{Extp}_{T_{1}}(0)$ has been obtained by M.G. Krein [25] (see also [1, 26] ). Other proofs are contained in [10, [21]. A generalization of the Krein result to the case of $C_{\mathfrak{H}}(\varphi)$-conractions, that is a description of the class $\operatorname{Extp}_{T_{1}}(\varphi)$ in the form (3.38) has been obtained in [5, 6] (see also [24, 14, 30, for other proofs).

3.5. A description of the set of all proper and improper $C_{\mathfrak{H}}(\varphi)$-extensions of symmetric contractions.

Let $A$ be a closed densely defined symmetric operator in $\mathfrak{H}$. It is known, that any $m$ dissipative (in particular selfadjoint) extension $\widetilde{A}$ of $A$ is a proper extension $\left(\widetilde{A} \in \operatorname{Extp}_{A}\right)$, that is $A \subset \widetilde{A} \subset A^{*}$. It is not the case for $m$-sectorial extensions of a nonnegative operator $A \geq 0$.

Therefore we clarify Definition 2.11 for the case of a nonnegative operator.

Definition 3.13. Let $A(\geq 0)$ be a closed densely defined nonnegative operator in $\mathfrak{H}$. Denote by $\operatorname{Ext}_{A}((0, \infty) ; \varphi)$ the class of all proper $m$-sectorial extensions of $A$ with vertex zero and half-angle $\varphi \in(0 ; \pi / 2)$. The class of all (proper and improper) $m$-sectorial extensions $\widetilde{A}(\in$ $\mathcal{C}(\mathfrak{H}))$ of $A$ will be denoted by $\operatorname{Extp}_{A}((0, \infty) ; \varphi)$. 
Here we present a description of the set $\operatorname{Ext}_{A}((0, \infty) ; \varphi)$. In accordance with the approach accepted in this paper (cf. Lemma 2.12) it suffices to describe the set $\operatorname{Ext}_{T_{1}}(\varphi)$ of all the extensions of the class $C_{T_{1}}(\varphi)$ of a nondensely defined Hermitian contraction $T_{1}:=$ $(I-A)(I+A)^{-1}\left(\in\left[\mathfrak{H}_{1}, \mathfrak{H}\right]\right)$ where $\mathfrak{H}_{1}:=\operatorname{ran}(I-A)$. In turn, considering the block-matrix representation of $T_{1}$ with respect to the orthogonal decomposition $\mathfrak{H}=\mathfrak{H}_{1} \oplus \mathfrak{H}_{2}$, one reduces the problem to the problem of a description of all the "completions" of a contractive operatormatrix $T_{1}=\left(\begin{array}{l}T_{11} \\ T_{21}\end{array}\right)$ to form a matrix $T=\left(T_{i j}\right)_{i, j=1}^{2} \in C_{\mathfrak{H}}(\varphi)$.

Note that $\widetilde{A} \in \operatorname{Extp}_{A}$ iff the entries $T_{12}$ and $T_{21}$ of $\left(T_{i j}\right)_{i, j=1}^{2}:=T:=(I-\widetilde{A})(I+\widetilde{A})^{-1}$ are connected by $T_{21}=T_{12}^{*}$.

Theorem 3.14. Let $T_{1}=\left(\begin{array}{c}T_{11} \\ T_{21}\end{array}\right)=\left(\begin{array}{c}T_{11}^{*} \\ V D_{T_{11}}^{*}\end{array}\right)$ be a symmetric contraction in $\mathfrak{H}=\mathfrak{H}_{1} \oplus \mathfrak{H}_{2}$, $T:=\left(T_{i j}\right)_{i, j=1}^{2} \in[\mathfrak{H}], \mathcal{H}_{2}:=\overline{\operatorname{ran}} D_{V^{*}}, \quad \mathcal{H}_{2}^{\prime}:=\overline{\operatorname{ran}} D_{V}$, and $\varphi \in[0, \pi / 2]$. Then

(i) $T \in \operatorname{Ext}_{T_{1}}(\pi / 2)$, i.e. $T\left(\in C_{\mathfrak{H}}(\pi / 2)\right)$ is a contractive extension of $T_{1}$ if and only if it is of the form (2.12), that is

$$
T_{12}=D_{T_{11}} U, \quad T_{22}=-V T_{11}^{*} U+D_{V^{*}} K D_{U} \quad \text { with } \quad U, K \in C(\pi / 2) .
$$

(ii) $T \in \operatorname{Ext}_{T_{1}}(\varphi):=\operatorname{Ext}_{T_{1}}(\pi / 2) \cap C_{\mathfrak{H}}(\varphi)$ if and only if $U$ "runs through" the operator ball of the form

$$
\begin{aligned}
U=\sin \varphi & \left(\sin ^{2} \varphi D_{V}^{2}+\cos ^{2} \varphi\right)^{-1 / 2} D_{V} M D_{V^{*}}\left(\sin ^{2} \varphi D_{V^{*}}^{2}+\cos ^{2} \varphi\right)^{-1 / 2}+ \\
& +\cos ^{2} \varphi\left(\sin ^{2} \varphi D_{V}^{2}+\cos ^{2} \varphi\right)^{-1} V^{*}, \quad M \in C(\pi / 2) \cap\left[\mathcal{H}_{2}, \mathcal{H}_{2}^{\prime}\right],
\end{aligned}
$$

and $T_{22}$ (for fixed $U$ ) "runs through" the operator "hole"

$$
T_{22}=-V T^{*} U+D_{V^{*}} K D_{U}, \quad K \sin \varphi \pm i Q \cos \varphi \in C(\pi / 2) \cap\left[\mathcal{H}_{1}, \mathcal{H}_{2}\right] .
$$

Here $\mathcal{H}_{1}:=\mathcal{H}_{1}(U):=\overline{\operatorname{ran}} D_{U}, Q=\bar{Q}_{0}\left(\in\left[\mathcal{H}_{1}, \mathcal{H}_{2}\right]\right)$ and $Q_{0}$ is defined by (3.11).

Proof. Equality (3.39) is implied by Theorem 2.4.

Let further $T_{12}=D_{T_{11}} U, T_{2}=\left(\begin{array}{c}T_{11} \\ U^{*} D_{T_{11}}\end{array}\right)$. Then $\left\{T_{1}, T_{2}\right\}$ is a dual pair of contractions and according to Theorem 3.4 the condition $\operatorname{Ext}_{\left\{T_{1}, T_{2}\right\}}(\varphi) \neq \varnothing$ is equivalent to the contractibility of the operator $Q_{0} \cos \varphi$, where $Q_{0}$ is defined by (3.11), i.e. to the inequality

$$
\cos \varphi \cdot\left\|D_{V^{*}}^{-1}(I-V U) f\right\| \leq\left\|D_{U} f\right\|, \quad f \in \mathfrak{H}_{2} .
$$

Supposing first that $0 \in \rho\left(D_{V^{*}}\right)$, we rewrite inequality (3.42) in the equivalent form

$$
\cos ^{2} \varphi\left[D_{V^{*}}^{-2}-U^{*} V^{*} D_{V^{*}}^{-2}-D_{V^{*}}^{-2} V U+U^{*} V^{*} D_{V^{*}}^{-2} V U\right] \leq I-U^{*} U
$$

or

$$
\begin{aligned}
U^{*}\left(1+\cos ^{2} \varphi V^{*} D_{V^{*}}^{-2} V\right) U-\cos ^{2} \varphi & \left(U^{*} D_{V}^{-2} V^{*}+V D_{V}^{-2} U\right) \\
& +\cos ^{2} \varphi D_{V^{*}}^{-2}-I \leq 0
\end{aligned}
$$

Since inequality (3.43) is equivalent to (3.42) the set of its solutions is nonempty for any fixed $V$. By Lemma 2.3 for any fixed $V$ the set of solutions of inequality (3.43), that is the set of operators $U$ obeying (3.43) , forms an operator ball $B\left(C_{0} ; R_{l}, R_{r}\right)$. Applying Lemma 2.3 we find its center and radii. We have

$$
\begin{aligned}
R_{l} & =Q_{1}^{-1 / 2}=\left(I+\cos ^{2} \varphi V^{*} D_{V^{*}}^{-2} V\right)^{-1 / 2}=\left(I+\cos ^{2} \varphi V^{*} V D_{V}^{-2}\right)^{-1 / 2} \\
& =\left[\cos ^{2} \varphi\left(D_{V}^{-2}-I\right)+I\right]^{-1 / 2}=\left[D_{V}^{-2}\left(\sin ^{2} \varphi D_{V}^{2}+\cos ^{2} \varphi\right)\right]^{-1 / 2} \\
& =\left(\sin ^{2} \varphi D_{V}^{2}+\cos ^{2} \varphi\right)^{-1 / 2} D_{V}
\end{aligned}
$$




$$
\begin{aligned}
C_{0} & =-Q_{1}^{-1} Q_{2}=-R_{l}^{2} Q_{2}=\cos ^{2} \varphi\left(\sin ^{2} \varphi D_{V}^{2}+\cos ^{2} \varphi\right)^{-1} D_{V}^{2} D_{V}^{-2} V^{*} \\
& =\cos ^{2} \varphi V^{*}\left(\sin ^{2} \varphi D_{V^{*}}^{2}+\cos ^{2} \varphi\right)^{-1}
\end{aligned}
$$

And finally

$$
\begin{aligned}
R_{r}^{2}= & Q_{2}^{*} Q_{1}^{-1} Q_{2}-Q_{3}=-Q_{2}^{*} C_{0}-Q_{3} \\
= & \cos ^{4} \varphi V D_{V}^{-2} V^{*}\left(\sin ^{2} \varphi D_{V^{*}}^{2}+\cos ^{2} \varphi\right)^{-1} I-\cos ^{2} \varphi D_{V^{*}}^{-2} \\
= & \cos ^{4} \varphi\left(I-D_{V^{*}}^{2}\right) D_{V^{*}}^{-2}\left(\sin ^{2} \varphi D_{V^{*}}^{2}+\cos ^{2} \varphi\right)^{-1}+I-\cos ^{2} \varphi D_{V^{*}}^{-2} \\
= & \left(\sin ^{2} \varphi D_{V^{*}}^{2}+\cos ^{2} \varphi\right)^{-1} \cdot \\
& \quad \cdot\left[\cos ^{4} \varphi D_{V^{*}}^{-2}-\cos ^{4} \varphi \cdot I+\left(I-\cos ^{2} \varphi D_{V^{*}}^{-2}\right)\left(\sin ^{2} \varphi D_{V^{*}}^{2}+\cos ^{2} \varphi\right)\right] \\
= & \left(\sin ^{2} \varphi D_{V^{*}}^{2}+\cos ^{2} \varphi\right)^{-1}\left[\sin ^{2} \varphi D_{V^{*}}^{2}+\cos ^{2} \varphi\left(1-\cos ^{2} \varphi-\sin ^{2} \varphi\right)\right] \\
= & \sin ^{2} \varphi\left(\sin ^{2} \varphi D_{V^{*}}+\cos ^{2} \varphi \cdot I\right)^{-1} D_{V^{*}}^{2} .
\end{aligned}
$$

Thus,

$$
R_{r}=\sin \varphi\left(\sin ^{2} \varphi D_{V^{*}}+\cos ^{2} \varphi \cdot I\right)^{-1 / 2} D_{V^{*}}
$$

Applying Lemma 2.3 and taking relations (3.44)-(3.46) into account we get that inequality (3.43) or, what is the same, inequality (3.42) is satisfied iff $U$ admits a representation (3.40) with some $M \in C(\pi / 2)$. Thus, we proved (3.40) under the additional assumption $0 \in$ $\rho\left(D_{V^{*}}\right)\left(\Longleftrightarrow 0 \in \rho\left(D_{V}\right)\right)$.

Next, we may easily free ourselves of the additional assumption $0 \in \rho\left(D_{V^{*}}\right)$ by passing to the limit. Actually since $D_{V^{*}}^{-1}>D_{r V^{*}}^{-1}, \quad r \in(0,1)$, inequality (3.42) takes place if and only if for any $r \in(0,1)$ the inequality

$$
\cos \varphi\left\|D_{r V^{*}}^{-1}(I-V) f\right\| \leq\left\|D_{U} f\right\|, \quad f \in \mathfrak{H}_{2},
$$

holds true. Since $0 \in \rho\left(D_{r V}\right)$, then in accordance with what has been proved in the previous step inequality (3.47) (for fixed $r<1$ ) is equivalent to equality (3.40) with $D_{V}$ and $D_{V^{*}}$ repleaced by $D_{r V}$ and $D_{r V^{*}}$ respectively. In these equalities it is possible to pass to the limit as $r \uparrow 1$ (in the sence of strong convergence).

Now the relations (3.41) follow from Theorem 3.4.

According to Theorem 3.14 (see formulas (3.39)-(3.41) ) any extension $T \in \operatorname{Ext}_{T_{1}}(\varphi)$ is uniquely determined by a pair $\{M, K\}$ of "free" parameters. Denote the corresponding extension $T$ by $T_{M, K}$.

Next we denote by $\operatorname{Ext}^{e} T_{1}(\varphi)$ the set of extreme points of $\operatorname{Ext}_{T_{1}}(\varphi)$.

Corollary 3.15. Let $T=T_{M, K} \in \operatorname{Ext}_{T_{1}}(\varphi)$. Then

(i) $T_{M, K} \in \operatorname{Ext}_{T_{1}}^{e}(\varphi)$ if and only if $M$ is a maximal partial isometry from $\mathcal{H}_{2}$ to $\mathcal{H}_{2}^{\prime}$ and $K \in L^{e}(Q ; \varphi)$, where $L(Q ; \varphi)$ is defined by (3.25);

(ii) if $M$ is a maximal partial isometry, then the following implication holds

$$
\sigma(C)=\{ \pm 1\} \quad \text { and } \quad \operatorname{ran} D_{K, Q}=\overline{\operatorname{ran}} D_{K, Q} \Longrightarrow T_{M, K} \in \operatorname{Ext}_{\left\{T_{1}, T_{2}\right\}}^{e}(\varphi) .
$$

Proof. It follows from (3.39) and (3.40) that the mapping $\{M, K\} \rightarrow T_{M, K}$ preserves convexity: if the "free" parameters $\left\{M_{j}, K_{j}\right\}, j \in\{1,2\}$ and $\{M, K\}$ are connected by $M=$ $t M_{1}+(1-t) M_{2}$ and $K=t K_{1}+(1-t) K_{2}$ with $t \in(0,1)$, then $T_{M, K}=t T_{M_{1}, K_{1}}+(1-t) T_{M_{2}, K_{2}}$. To complete the proof it remains to apply both Proposition 2.7] and Corollary [3.10. 
Remark 3.16. (i) The set $\operatorname{Ext}_{T_{1}}(\varphi)$ of all (proper and improper) $C_{\mathfrak{H}}(\varphi)$-extensions of $T_{1}$ admits a representation $\operatorname{Ext}_{T_{1}}(\varphi)=\cup_{T_{2}} \operatorname{Ext}\left\{T_{1}, T_{2}\right\}(\varphi)$ where $T_{2}=\left(\begin{array}{c}T_{11} \\ U^{*} D_{T_{11}}\end{array}\right)$ and $U$ "runs through" the operator ball (3.40). Note that $T$ is a proper $C_{\mathfrak{H}}(\varphi)$-extension of $T_{1}$ iff $U=V^{*}$. In this case $Q=I$ and (3.41) turns into (3.36).

(ii) Theorem 3.14 has been proved by the author together with V. Kolmanovich in [24] in a different but equivalent form.

3.6. $C_{\mathfrak{H}}(\varphi)$-extensions of a dual pair of $C(\varphi)$-contractions. Here we consider a dual pair $\left\{T_{1}, T_{2}\right\}$ of $C(\varphi)$-contractions of the form

$$
T_{1}=\left(\begin{array}{c}
T_{11} \\
T_{21}
\end{array}\right)=\left(\begin{array}{c}
T_{11} \\
V D_{T_{11}}
\end{array}\right), \quad T_{2}=\left(\begin{array}{c}
T_{11}^{*} \\
T_{12}
\end{array}\right)=\left(\begin{array}{c}
T_{11}^{*} \\
U^{*} D_{T_{11}^{*}}
\end{array}\right),
$$

and show that the Problem $2 C$ mentioned in the Introduction is reduced to the Problem 3 with different left $R_{l}^{+} \neq R_{l}^{-}$and right $R_{r}^{+} \neq R_{r}^{-}$radii.

Proposition 3.17. Let $\left\{T_{1}, T_{2}\right\}$ be a dual pair of conractions in $\mathfrak{H}=\mathfrak{H}_{1} \oplus \mathfrak{H}_{2}$. Suppose that both $T_{1}$ and $T_{2}$ obey condition (2.15) with $\varphi=\varphi_{0}$. Then

(i) for any $\varphi \in\left[\varphi_{0}, \pi / 2\right)$ the following relations hold

$$
2\left(T_{11}^{*}\right)_{I}=\tan \varphi \cdot D_{T_{11}} C(\varphi) D_{T_{11}}=\tan \varphi \cdot D_{T_{11}^{*}} C_{2}(\varphi) D_{T_{11}^{*}},
$$

where $C_{j}(\varphi):=C_{j} \cdot \tan \varphi_{0} / \tan \varphi, j \in\{1,2\}$, is a selfadjoint conraction and

$$
C_{1}=D_{V} C^{\prime} D_{V}, \quad C_{2}=D_{U^{*}} C^{\prime \prime} D_{U^{*}}, \quad-I \leq C^{\prime}, C^{\prime \prime} \leq+I
$$

(ii) for any $\varphi \in\left(\varphi_{0}, \pi / 2\right)$ the set $\operatorname{Ext}_{\left\{T_{1}, T_{2}\right\}}(\varphi)$ forms an operator hole:

$$
T \in \operatorname{Ext}_{\left\{T_{1}, T_{2}\right\}}(\varphi) \Longleftrightarrow T_{22} \sin \varphi \in L(\varphi):=B\left(C_{+} ; R_{l}^{+}, R_{r}^{+}\right) \cap B\left(C_{-} ; R_{l}^{-}, R_{r}^{-}\right),
$$

where

$$
C_{ \pm}:=\mp i \cos \varphi \cdot I-V_{ \pm}\left(T_{11}^{*} \sin \varphi \pm i \cos \varphi\right) U_{ \pm}, \quad R_{l}^{ \pm}=D_{V_{ \pm}^{*}}, R_{r}^{ \pm}=D_{U_{ \pm}}
$$

and $V_{ \pm}, U_{ \pm}$are contractions of the form

$$
V_{ \pm}^{*}:=V_{1 \pm}^{*}\left(I \pm C_{1}(\varphi)\right)^{-1 / 2} V^{*}, \quad U_{ \pm}:=U_{1 \pm}\left(I \pm C_{2}(\varphi)\right)^{-1 / 2} U
$$

and $V_{1 \pm}, U_{1 \pm}$ are (uniquely determined) partial isometries.

In particular, Ext $\left\{T_{1}, T_{2}\right\}(\varphi) \neq \emptyset$ if and only if $L(\varphi) \neq \emptyset$.

Proof. The inclusion $T=\left(T_{i j}\right)_{i, j=1}^{2} \in C_{\mathfrak{H}}(\varphi)$ means that

$$
T_{ \pm}:=\left(\begin{array}{ll}
B_{11}^{ \pm} & B_{12} \\
B_{21} & B_{2}^{ \pm}
\end{array}\right):=\left(\begin{array}{cc}
\sin \varphi \cdot T_{11} \pm i \cos \varphi \cdot I & T_{21} \sin \varphi \\
T_{21} \sin \varphi & \sin \varphi \cdot T_{22} \pm i \cos \varphi \cdot I
\end{array}\right) \in C_{\mathfrak{H}}(\pi / 2)
$$

First we note that

$$
D_{T_{1}}^{2}=I-T_{11}^{*} T_{11}-T_{21}^{*} T_{21}=D_{T_{11}}^{2}-D_{T_{11}} V^{*} V D_{T_{11}}=D_{T_{11}} D_{V}^{2} D_{T_{11}}
$$

and $D_{T_{2}}^{2}=D_{T_{11}^{*}} D_{U^{*}}^{2} D_{T_{11}^{*}}$. Combining these relations with (2.15) and applying Lemma 3.1] we obtain (3.49).

Next, starting with (3.54) and taking (3.49) into account we get

$$
\begin{array}{r}
D_{B_{11}^{ \pm}}^{2}=I-\left(\sin \varphi \cdot T_{11}^{*} \mp i \cos \varphi\right)\left(\sin \varphi \cdot T_{11} \pm i \cos \varphi\right) \\
=\sin ^{2} \varphi \cdot D_{T_{11}}^{2} \mp \sin 2 \varphi \cdot\left(T_{11}\right)_{I}=\sin ^{2} \varphi \cdot D_{T_{11}}\left(I \pm C_{1}(\varphi)\right) D_{T_{11}} .
\end{array}
$$


Hence there exist partial isometries $V_{1 \pm}(\varphi)$ such that

$$
D_{B_{11}^{ \pm}}=\sin \varphi \cdot D_{T_{11}}\left(I \pm C_{1}(\varphi)\right)^{1 / 2} V_{1 \pm}(\varphi)=\sin \varphi \cdot V_{1 \pm}^{*}(\varphi)\left(I \pm C_{1}\right)^{1 / 2} D_{T_{11}}
$$

Combining (3.54), (3.55) and (3.56) we derive

$$
B_{21}=T_{21} \sin \varphi=\sin \varphi \cdot V D_{T_{11}}=V_{ \pm} D_{B_{11}^{ \pm}}=\sin \varphi \cdot V_{ \pm} V_{1 \pm}^{*}(\varphi)\left(I \pm C_{1}\right)^{1 / 2} \cdot D_{T_{11}}
$$

It follows that $V=V_{ \pm} \cdot V_{1 \pm}^{*}(\varphi)\left(I \pm C_{1}(\varphi)\right)^{1 / 2}$, which yields the first of relations (3.53).

Similarly we get

$$
D_{B_{11}^{ \pm *}}^{2}=\sin ^{2} \varphi \cdot D_{T_{11}^{*}}\left(I \pm C_{2}(\varphi)\right) D_{T_{11}^{*}} \cdot
$$

According to polar decomposition we have $\sin \varphi \cdot\left(I \pm C_{2}(\varphi)\right)^{1 / 2} D_{T_{11}^{*}}=U_{1 \pm} D_{B_{11}^{ \pm *}}$ with some partial isometries $U_{ \pm}$. These representations imply

$$
B_{12}=T_{12} \sin \varphi=\sin \varphi \cdot D_{T_{11}^{*}} U=D_{B_{11}^{ \pm *}} U_{ \pm}=\sin \varphi \cdot D_{T_{11}^{*}}\left(I \pm C_{2}(\varphi)\right)^{1 / 2} U_{1 \pm} U_{ \pm} .
$$

Hence $U=\left(I \pm C_{2}(\varphi)\right)^{1 / 2} U_{1 \pm} U_{ \pm}$. This equality yields the second relation in (3.53).

By Theorem 2.4 $T_{ \pm} \in C_{\mathfrak{H}}(\pi / 2)$ if and only if

$$
T_{22} \sin \varphi \pm i \cos \varphi \cdot I=C_{ \pm}^{\prime}+D_{V_{ \pm}^{*}} K_{ \pm} D_{U_{ \pm}}
$$

where $K_{ \pm}$are contractions and $C_{ \pm}^{\prime}$ are defined by

$$
C_{ \pm}^{\prime}=-V_{ \pm}\left(B_{11}^{ \pm}\right)^{*} U_{ \pm}=-V_{ \pm}\left(T_{11}^{*} \sin \varphi \pm i \cos \varphi \cdot I\right) U_{ \pm} .
$$

to complete the proof it suffices to set $C_{ \pm}=C_{ \pm}^{\prime} \mp i \cos \varphi \cdot I$.

Remark 3.18. Let $T_{1}=\left(\begin{array}{l}T_{11} \\ T_{21}\end{array}\right) \in C(\varphi)$ and $\mathfrak{H}=\mathfrak{H}_{1} \oplus \mathfrak{H}_{2}$. It is shown in 30], Theorem 4.11, that Problem 3C is reduced to Problem 5 mentioned in the Introduction. Namely, it is proved in [30] that $T \in \operatorname{Ext}_{T_{1}}(\varphi)$ iff

$$
T P_{2} \in L:=B_{+} \cap B_{-}, \quad \text { where } \quad B_{ \pm}=B\left(C_{ \pm} ; D_{S_{ \pm}^{*}} / \sin \varphi, P_{2}\right),
$$

and $S_{ \pm}=T_{1} \sin \varphi \pm i \cos \varphi \cdot I, \quad C_{ \pm}=\mp \cot \varphi \cdot P_{2}$. Thus, the set $\operatorname{Ext}_{T_{1}}(\varphi)$ forms an operator hole of the form (1.5) with $R_{l}^{ \pm}=D_{S_{ \pm}^{*}} / \sin \varphi, R_{r}^{ \pm}=P_{2}$, and $C_{ \pm}=\mp \cot \varphi \cdot P_{2}$.

\section{Noncontractive EXtensions of DUAL PAIR OF SYMMETRIC CONTRACTiOns.}

4.1. Schur complements. In this section we investigate some spectral properties of contractive and noncontractive extensions of a dual pair $\left\{T_{1}, T_{2}\right\}$ of symmetric contractions using their block-matrix representations (3.9). Trough this section we keep a notation $T_{K}$ for any (not necessary contractive) extension of the dual pair $\left\{T_{1}, T_{2}\right\}$ having the form (2.12) with a bounded operator $K \in\left[\mathcal{H}_{1}, \mathcal{H}_{2}\right]$. Observe that any bounded extension $T \in \operatorname{Ext}_{\left\{T_{1}, T_{2}\right\}}$ has such a form iff $T_{1}$ and $T_{2}$ are transversal, that is $0 \in \rho\left(D_{U}\right) \cap \rho\left(D_{V^{*}}\right)$. Note also that in the nonsingular case $\mathcal{H}_{1}=\mathfrak{H}_{1}$ and $\mathcal{H}_{2}=\mathfrak{H}_{2}^{\prime}$.

We investigate some spectral properties of extensions $T_{K}\left(\in \operatorname{Ext}_{\left\{T_{1}, T_{2}\right\}}\right)$ in terms of "boundary" operators $K$. In particular we obtain descriptions of the classes $C_{\mathfrak{H}}\left(\varphi ; \varkappa^{ \pm}\right)$and $C_{\mathfrak{H}}\left(\varphi ; \mathfrak{S}^{ \pm}\right)$.

As well as in Theorem 3.14 these descriptions essentially depend on the operator

$$
Q_{0}:=D_{V^{*}}^{-1}(I-V U) D_{U}^{-1} \text {. }
$$

In the following theorem which is the main result of the section we calculate Schur complement of the operator block-matrices $I-T_{K} T_{K}^{*} \pm \cot \varphi \cdot\left(T_{K}-T_{K}^{*}\right)$. 
Theorem 4.1. Let $\left\{T_{1}, T_{2}\right\}$ be a dual pair of symmetric contractions of the form (3.9) in $\mathfrak{H}=\mathfrak{H}_{1} \oplus \mathfrak{H}_{2}, \quad T_{K} \in \operatorname{Ext}_{\left\{T_{1}, T_{2}\right\}}$ and let $\varphi \in\left[\varphi_{1}, \pi / 2\right]$ where $\varphi_{1}:=\arccos \left(\|Q\|^{-1}\right), \quad Q:=\bar{Q}_{0}$ and $Q_{0}$ be defined by (4.1). Let further

$$
\begin{aligned}
& S^{ \pm}:=\left(S_{i j}^{ \pm}\right)_{i, j=1}^{2}:=I-T_{K} T_{K}^{*} \pm \cot \varphi \cdot\left(T_{K}-T_{K}^{*}\right), \\
& G^{ \pm}:=\left(G_{i j}^{ \pm}\right)_{i, j=1}^{2}:=I-T_{K}^{*} T_{K} \pm \cot \varphi \cdot\left(T_{K}-T_{K}^{*}\right),
\end{aligned}
$$

with $S_{i j}^{ \pm}, G_{i j}^{ \pm} \in\left[\mathfrak{H}_{i}, \mathfrak{H}_{j}\right]$. Then

(i) $\operatorname{ran}\left(S_{11}^{1 / 2}\right) \supset \operatorname{ran}\left(S_{12}^{ \pm}\right), \operatorname{ran}\left(G_{11}^{1 / 2}\right) \supset \operatorname{ran}\left(G_{12}^{ \pm}\right)$and, consequently the operators $S_{11}^{-1 / 2} S_{12}^{ \pm}$ and $G_{11}^{-1 / 2} G_{12}^{ \pm}$are well defined and bounded, where $S_{11}:=S_{11}^{+}=S_{11}^{-}$;

(ii) the identities

$$
\begin{array}{cl}
\sin ^{2} \varphi & \cdot\left[S_{22}^{ \pm}-\left(S_{11}^{-1 / 2} S_{12}^{ \pm}\right)\left(S_{11}^{-1 / 2} S_{12}^{ \pm}\right)^{*}\right] \\
= & D_{V^{*}} \cdot\left[I-(K \sin \varphi \mp i Q \cos \varphi)\left(K^{*} \sin \varphi \pm i Q^{*} \cos \varphi\right)\right] \cdot D_{V^{*}}, \\
\sin ^{2} \varphi & \cdot\left[G_{22}^{ \pm}-\left(G_{11}^{-1 / 2} G_{12}^{ \pm}\right)^{*}\left(G_{11}^{-1 / 2} G_{12}^{ \pm}\right)\right] \\
= & D_{U} \cdot\left[I-\left(K^{*} \sin \varphi \pm i Q^{*} \cos \varphi\right)(K \sin \varphi \mp i Q \cos \varphi)\right] \cdot D_{U},
\end{array}
$$

hold true.

Proof. (i) We let $\left(G_{i j}\right)_{i, j=1}^{2}:=I-T_{K}^{*} T_{K}$. Then

$$
\left(G_{i j}^{ \pm}\right)_{i, j=1}^{2}=\left(G_{i j}\right)_{i, j=1}^{2} \pm i \cot \varphi\left(\begin{array}{cc}
0 & D_{T_{11}}\left(U-V^{*}\right) \\
\left(V-U^{*}\right) D_{T_{11}} & T_{22}-T_{22}^{*}
\end{array}\right) .
$$

By definition the operator $T_{K}\left(\in \operatorname{Ext}_{\left\{T_{1}, T_{2}\right\}}\right)$ is of the form (2.12) with $T_{22}=-V T_{11} U+$ $D_{V^{*}} K D_{U}$ and $K \in\left[\mathcal{H}_{1}, \mathcal{H}_{2}\right]$. Therefore taking into account (2.12) and (4.6) we get

$$
G_{11}^{ \pm}=G_{11}=D_{T_{11}} D_{V}^{2} D_{T_{11}}
$$

and

$$
\left.-G_{21}^{ \pm}=-\left(G_{12}\right)^{ \pm}\right)^{*}=\left(U^{*} T_{11} D_{V}+D_{U} K^{*} V\right) D_{V} D_{T_{11}} \mp i \cot \varphi \cdot\left(U^{*}-V\right) D_{T_{11}} .
$$

Since $\varphi \in\left[\varphi_{1}, \pi / 2\right]$, then according to Theorem 3.14 the operators $U$ and $V$ are connected by equality (3.40). Setting

$$
Y:=\left(\sin ^{2} \varphi D_{V}^{2}+\cos ^{2} \varphi\right)^{-1 / 2} \text { and } Y_{*}=\left(\sin ^{2} \varphi D_{V^{*}}^{2}+\cos ^{2} \varphi\right)^{-1 / 2},
$$

and taking into account the identity $V D_{V}=D_{V^{*}} V$ we rewrite (3.40) in the form

$$
U^{*}-V=\sin \varphi D_{V^{*}} Y_{*} \cdot\left(M^{*}-\sin \varphi \cdot V\right) \cdot Y D_{V} .
$$

Let, further

$$
X_{ \pm}:=U^{*} T_{11} D_{V}+D_{U} K^{*} V \mp i \cos \varphi \cdot D_{V^{*}} Y_{*} \cdot\left(M^{*}-\sin \varphi \cdot V\right) \cdot Y .
$$

Now relations (4.8)-(4.11) yield $-G_{21}^{ \pm}=X_{ \pm} D_{V} D_{T_{11}}$. Combining this equality with (4.7) we easily get

$$
\left\|G_{12}^{ \pm} f\right\|^{2} \leq\left\|X_{ \pm}\right\|^{2} \cdot\left(G_{11} f, f\right)=\left\|X_{ \pm}\right\|^{2} \cdot \|\left(G_{11}^{1 / 2} f \|^{2}, \quad f \in \mathfrak{H}_{1} .\right.
$$

This inequality yields the inclusion $\operatorname{ran}\left(G_{12}^{ \pm}\right) \subset \operatorname{ran}\left(G_{11}^{1 / 2}\right)$, that is the second of the required inclusions. 
The proof of the first inclusion $\operatorname{ran}\left(S_{11}^{12}\right) \supset \operatorname{ran}\left(S_{12}^{ \pm}\right)$can be obtained in just the same way. It is suffices to use the equalities $S_{11}^{ \pm}=S_{11}=D_{T_{11}} D_{U^{*}}^{2} D_{T_{11}}$ and $S_{21}^{ \pm}=X_{ \pm}^{\prime} D_{U^{*}} D_{T_{11}}$ in place of (4.7) and (4.11) respectively, and the relation

$$
\begin{aligned}
V-U^{*}=\sin \varphi \cdot D_{U}\left(\sin ^{2} \varphi D_{U}^{2}+\right. & \left.\cos ^{2} \varphi\right)^{-1 / 2} \\
& \left(M_{1}-\sin \varphi \cdot U^{*}\right) \cdot\left(\sin ^{2} \varphi D_{U^{*}}^{2}+\cos ^{2} \varphi\right)^{-1 / 2} D_{U^{*}}
\end{aligned}
$$

in place of (4.10).

$\left(i i_{1}\right)$ Let us prove equality (4.5) assuming at the begining that $0 \in \rho\left(D_{T_{11}}\right) \cap \rho\left(D_{V}\right)$. In this case setting $Z:=T_{22}$, we obtain from (4.6) - (4.8) that

$$
\begin{aligned}
& G_{22}^{ \pm}-\left(G_{12}^{ \pm}\right)^{*} G_{11}^{-1} G_{12}^{ \pm}=I-U^{*} D_{T_{11}}^{2} U-Z^{*} Z \pm i \cot \varphi \cdot\left(Z-Z^{*}\right) \\
& -\left[U^{*}\left(T_{11} \pm i \cot \varphi\right) \pm\left(Z^{*} \mp i \cot \varphi\right) V\right] \cdot D_{V}^{-2} \cdot\left[\left(T_{11} \mp i \cot \varphi\right) U+V^{*}(Z \pm i \cot \varphi)\right] \\
& =\left(1+\cot ^{2} \varphi\right) \cdot I-U^{*} D_{T_{11}}^{2} U-\left(Z^{*} \mp i \cot \varphi \cdot I\right) \cdot(Z \pm i \cot \varphi \cdot I) \\
& -\left(Z^{*} \mp i \cot \varphi\right) \cdot V D_{V}^{-2} V^{*} \cdot(Z \pm i \cot \varphi)-U^{*}\left(T_{11} \pm i \cot \varphi\right) \cdot D_{V}^{-2} V^{*} \cdot(Z \pm i \cot \varphi) \\
& -\left(Z^{*} \mp i \cot \varphi\right) \cdot V D_{V}^{-2} \cdot\left(T_{11} \mp i \cot \varphi\right) U-U^{*}\left(T_{11} \pm i \cot \varphi\right) \cdot D_{V}^{-2} \cdot\left(T_{11} \mp i \cot \varphi\right) U \\
& =D_{U}^{2}+\cot ^{2} \varphi \cdot I+U^{*} T_{11}^{2} U-\left(Z^{*} \mp i \cot \varphi\right) \cdot D_{V^{*}}^{-2} \cdot(Z \pm i \cot \varphi \cdot I) \\
& -U^{*}\left(T_{11} \pm i \cot \varphi\right) \cdot D_{V}^{-2} V^{*} \cdot(Z \pm i \cot \varphi)-\left(Z^{*} \mp i \cot \varphi\right) \cdot V D_{V}^{-2} \cdot\left(T_{11} \mp i \cot \varphi\right) U \\
& -U^{*}\left(T_{11} \pm i \cot \varphi\right) \cdot D_{V}^{-2} \cdot\left(T_{11} \mp i \cot \varphi\right) U .
\end{aligned}
$$

On the other hand, combining (4.1) with the equality $Z:=T_{22}=-V T_{11} U+D_{V^{*}} K D_{U}$, we get

$$
Q D_{U}=D_{V^{*}}^{-1}(I-V U) \quad \text { and } \quad K D_{U}=D_{V^{*}}^{-1}\left(Z+V T_{11} U\right) .
$$

Inserting these relations in the right-hand side of (4.5) we deduce

$$
\begin{aligned}
& A_{\mp}:=D_{U} \cdot\left[\frac{1}{\sin ^{2} \varphi}-\left(K^{*} \pm i Q^{*} \cot \varphi\right) \cdot(K \mp i Q \cot \varphi)\right] \cdot D_{U} \\
& =\frac{D_{U}^{2}}{\sin ^{2} \varphi}-\left[\left(Z^{*}+U^{*} T_{11} V^{*}\right) \mp i \cot \varphi\left(I-U^{*} V^{*}\right)\right] \cdot D_{V^{*}}^{-2} \cdot\left[\left(Z+V T_{11} U\right) \pm i \cot \varphi(I-V U)\right] \\
& =\frac{D_{U}^{2}}{\sin ^{2} \varphi}-\left[\left(Z^{*} \mp i \cot \varphi\right)+U^{*}\left(T_{11} \pm i \cot \varphi\right) V^{*}\right] \cdot D_{V^{*}}^{-2} \cdot\left[(Z \pm i \cot \varphi)+V\left(T_{11} \mp i \cot \varphi\right) U\right] \\
& =\frac{D_{U}^{2}}{\sin ^{2} \varphi}-\left(Z^{*} \mp i \cot \varphi\right) \cdot D_{V^{*}}^{-2} \cdot(Z \pm i \cot \varphi)-\left(Z^{*} \mp i \cot \varphi\right) \cdot D_{V^{*}}^{-2} V \cdot\left(T_{11} \mp i \cot \varphi\right) U \\
& -U^{*}\left(T_{11} \pm i \cot \varphi\right) \cdot V^{*} D_{V^{*}}^{-2} \cdot(Z \pm i \cot \varphi)-U^{*}\left(T_{11} \pm i \cot \varphi\right) \cdot V^{*} D_{V^{*}}^{-2} V \cdot\left(T_{11} \mp i \cot \varphi\right) U
\end{aligned}
$$

Since $V^{*} D_{V^{*}}^{-2} V=D_{V}^{-2} V^{*} V=D_{V}^{-2}-I$, then the last term in (4.16) is transformed as follows:

$$
\begin{aligned}
& -U^{*}\left(T_{11} \pm i \cot \varphi\right) \cdot V^{*} D_{V^{*}}^{-2} V \cdot\left(T_{11} \mp i \cot \varphi\right) U=U^{*} T_{11}^{2} U \\
& +\cot ^{2} \varphi \cdot U^{*} U-U^{*}\left(T_{11}-i \cot \varphi\right) \cdot D_{V}^{-2} \cdot\left(T_{11}+i \cot \varphi\right) U
\end{aligned}
$$


Comparing (4.14) with (4.16) and (4.17) and noting that

$$
\frac{1}{\sin ^{2} \varphi} D_{U}^{2}+\cot ^{2} \varphi \cdot U^{*} U=D_{U}^{2}+\cot ^{2} \varphi \cdot\left(D_{U}^{2}+U^{*} U\right)=D_{U}^{2}+\cot ^{2} \varphi \cdot I,
$$

we arrive at the equality

$$
A_{\mp}=G_{22}^{ \pm}-\left(G_{11}^{-1 / 2} G_{12}^{ \pm}\right)^{*}\left(G_{11}^{-1 / 2} G_{12}^{ \pm}\right)
$$

coinciding with (4.5).

$\left(i i_{2}\right)$ Now we free ourselves of the additional restriction $0 \in \rho\left(D_{V^{*}}\right) \cap \rho\left(D_{T_{11}}\right)$. Consider the strict contractions $r T_{1}=\left(\begin{array}{c}r T_{11} \\ r T_{21}\end{array}\right), r \in(0,1)$. We have $r T_{21}=V(r) D_{r T_{11}}$, where

$$
V(r):=r V D_{T_{11}} D_{r T_{11}}^{-1}=r V\left(I-T_{11}^{*} T_{11}\right)^{1 / 2} \cdot\left(I-r^{2} T_{11}^{*} T_{11}\right)^{-1 / 2} .
$$

Let us define the operator $U(r)$ by (3.40) with $V$ replaced by $V(r)$, but not replacing $M$. Then the operator

$$
Q_{0}(r):=D_{V^{*}(r)}^{-1} \cdot(I-V(r) U(r)) \cdot D_{U(r)}^{-1}
$$

is bounded and $Q_{0}(r) \cos \varphi$ is contractive. We set $Q(r):=\overline{Q_{0}(r)}$ and note that $Q(r) \cos \varphi \in$ $C(\pi / 2)$.

Next, starting with $U(r)$ we define a dual pair of Hermitian contractions $\left\{r T_{1}, T_{2}(r)\right\}$ by setting

$$
T_{21}(r):=D_{r T_{11}} U(r) \quad \text { and } \quad T_{2}(r):=\left(\begin{array}{c}
r T_{11} \\
T_{21}^{*}(r)
\end{array}\right) .
$$

Denote by $T_{K}(r)\left(\in \operatorname{Ext}_{\left\{r T_{1}, T_{2}(r)\right\}}\right)$ the extension of $\left\{T_{1}, T_{2}\right\}$ defined by the same operator $K$, as the extension $T_{K} \in \operatorname{Ext}_{\left\{T_{1}, T_{2}\right\}}$, that is

$$
T_{K}(r):=\left(\begin{array}{ll}
r T_{11} & T_{12}(r) \\
r T_{21} & T_{22}(r)
\end{array}\right), \quad T_{22}(r):=-r V(r) T_{11} U(r)+D_{V^{*}(r)} K D_{U(r)} .
$$

Since $0 \in \rho\left(D_{r T_{11}}\right) \cap \rho\left(D_{V(r)}\right)$ and $Q(r) \cos \varphi \in C(\pi / 2)$, then for the operator-matrix

$$
\left(G_{i j}^{ \pm}(r)\right)_{i, j=1}^{2}:=I-T_{K}^{*}(r) T_{K}(r) \pm i \cot \varphi\left(T_{K}(r)-T_{K}^{*}(r)\right)
$$

equality (4.5) is already proved in the previous step, that is

$$
\begin{aligned}
& \sin ^{2} \varphi \cdot\left[G_{22}^{ \pm}(r)-\left(G_{12}^{ \pm}(r)\right)^{*} G_{11}^{-1}(r) G_{12}(r)\right] \\
& =D_{U(r)} \cdot\left[I-\left(K^{*} \sin \varphi \pm i Q^{*}(r) \cos \varphi\right) \cdot(K \sin \varphi \mp i Q(r) \cos \varphi)\right] \cdot D_{U(r)} .
\end{aligned}
$$

It remains to justify the possibility to pass to the limit in (4.22) as $r \rightarrow 1$. We may assume without rstriction of generality that $\operatorname{ker} G_{11}=\{0\}$. Then, as it follows from (4.7), (4.8) and (4.11),

$$
G_{11}^{1 / 2}=U_{1} D_{V} D_{T_{11}}=D_{T_{11}} D_{V} U_{1}^{*}, \quad G_{11}^{-1 / 2} G_{12}^{ \pm}=-U_{1} X_{ \pm}^{*}, \quad\left(G_{11}^{-1 / 2} G_{12}^{ \pm}\right)^{*}=-X_{ \pm} U_{1}^{*}
$$

where the operator $U_{1}$ is unitary.

Further, introducing the operators

$$
Y(r):=\left(\sin ^{2} \varphi \cdot D_{V(r)}^{2}+\cos ^{2} \varphi\right)^{-1 / 2} \quad \text { and } \quad Y_{*}(r):=\left(\sin ^{2} \varphi \cdot D_{V^{*}(r)}+\cos ^{2} \varphi\right)^{-1 / 2}
$$

one derives from the definition of the operator $U^{*}(r)(r<1)$ that

$$
U^{*}(r)-V(r)=\sin \varphi D_{V^{*}(r)} Y_{*}(r) \cdot\left(M^{*}-r \sin \varphi \cdot V(r)\right) \cdot Y(r) D_{V(r)} .
$$


Next, we define $G_{11}^{1 / 2}(r)$ and $G_{21}^{ \pm}(r)$ by (4.7) and (4.8) with $V(r), U(r)$ and $r T_{11}$ in place of $V, U$ and $T_{11}$ respectively. Further, similarly to definition (4.11) of $X_{ \pm}$we set $(4.26)$

$$
X_{ \pm}(r):=r U^{*}(r) T_{11} D_{V(r)}+D_{U(r)} K^{*} V(r) \mp i \cos \varphi \cdot Y_{*}(r) D_{V *(r)}\left(M^{*}-r \sin \varphi \cdot V(r)\right) Y(r) .
$$

Combining these definitions we arrive at the relations

$$
G_{11}^{1 / 2}(r)=U_{1}(r) D_{V(r)} D_{r T_{11}} \quad \text { and } \quad G_{21}^{ \pm}(r)=X_{ \pm}(r) D_{V(r)} D_{r T_{11}},
$$

which are analogous to that of (4.23). Here $U_{1}(r), r \in(0,1)$, is a family of unitary operators. Hence

$$
-\left(G_{12}^{ \pm}(r)\right)^{*} G_{11}^{-1 / 2}(r)=X_{ \pm}(r) U_{1}^{*}(r), \quad-G_{11}^{-1 / 2} G_{12}^{ \pm}(r)=U_{1}(r) X_{ \pm}(r) .
$$

It follows from (4.18) that $s-\lim _{r \rightarrow 1} V(r)=V$ and $s-\lim _{r \rightarrow 1} V^{*}(r)=V^{*}$. Hence and taking into account (4.24) we get

$$
\begin{aligned}
s-\lim _{r \rightarrow 1} D_{V(r)} & =D_{V}, & s-\lim _{r \rightarrow 1} D_{V^{*}(r)} & =D_{V^{*}}, \\
s-\lim _{r \rightarrow 1} Y(r) & =Y, & s-\lim _{r \rightarrow 1} Y_{*}(r) & =Y_{*} .
\end{aligned}
$$

Relations (4.25), (4.29) and (4.10) yield

$$
s-\lim _{r \rightarrow 1} U(r)=U, \quad s-\lim _{r \rightarrow 1} U^{*}(r)=U^{*}, \quad s-\lim _{r \rightarrow 1} D_{U(r)}=D_{U} .
$$

It follows from (4.20) and (4.21) that

$$
s-\lim _{r \rightarrow 1} G_{22}(r)=G_{22}
$$

Further, (4.26) and (4.11) yield $s-\lim _{r \rightarrow 1} X_{ \pm}(r)=X_{ \pm}$and $s-\lim _{r \rightarrow 1} X_{ \pm}^{*}(r)=X_{ \pm}^{*}$. Therefore combining relations (4.22) with (4.28) and taking into account the obvious identities $U_{1}^{*}(r) U_{1}(r)=U_{1}^{*} U_{1}=I$ we arrive at

$$
s-\lim _{r \rightarrow 1}\left(G_{12}^{ \pm}(r)\right)^{*} G_{11}^{-1}(r) G_{12}^{ \pm}(r)=s-\lim _{r \rightarrow 1} X_{ \pm}(r) X_{ \pm}^{*}(r)=X_{ \pm} X_{ \pm}^{*}=\left(G_{11}^{-1 / 2} G_{12}^{ \pm}\right)^{*} G_{11}^{-1 / 2} G_{12}^{ \pm} .
$$

Relations (4.31) and (4.32) allow us to pass to the limit in left-hand side of (4.22) as $r \rightarrow 1$. So, it remains to justify passage to the limit in the right hand side of (4.22). In turn it suffices to prove the relations

$$
s-\lim _{r \rightarrow 1} Q(r) D_{U(r)}=Q D_{U} \quad \text { and } \quad s-\lim _{r \rightarrow 1} D_{U(r)} Q^{*}(r)=D_{U} Q^{*} .
$$

We derive from (4.25) and (4.19) that

$$
\begin{aligned}
Q(r) D_{U(r)} & =D_{V^{*}(r)}^{-1}(I-V(r) U(r)) \\
& =\left\{I-\sin \varphi Y(r) V(r)\left(M-r \sin \varphi \cdot V^{*}(r)\right) Y_{*}(r)\right\} D_{V^{*}(r)} .
\end{aligned}
$$

It follows from (4.29) that there exists the limit of the right-hand side of (4.34) as $r \rightarrow 1$. Hence there exist the limit of the left-hand side of (4.34) as $r \rightarrow 1$. Moreover, the first of relations (4.33) is now implied by (4.34) and similar formula for $Q D_{U}$ which follows from (4.10). The second formula in (4.33) may be proved similarly.

Finally, passing to the limit in (4.22) as $r \rightarrow 1$ and taking into account (4.31), (4.32) and (4.33) we arrive at (4.5). Relation (4.4) may be proved in just the same way. 
4.2. Descriptions of the classes $C\left(\pi / 2 ; \varkappa_{ \pm}\right)$and $T \in C\left(\pi / 2 ; \mathfrak{S}^{ \pm}\right)$

Here we present some corollaries from Theorem 4.1. To formulate them we need some definitions and an elementary lemma.

Let $\varkappa_{-}(\mathfrak{t})$ be the number of negative squares of the symmetris quadratic form $\mathfrak{t}$, that is the maximum dimensions of the "negative" linear manifolds

$$
L_{-}=\{f \in \mathcal{D}(\mathfrak{t}) \backslash\{0\}: \mathfrak{t}[f]<0\} \cup\{0\} .
$$

For any selfadjoint operator $T=T^{*} \in \mathcal{C}(\mathfrak{H})$ with the resolution of the identity $E_{T}(\cdot)$ we let $T_{-}:=E_{T}(-\infty, 0) T$ and $\varkappa_{-}(T):=\operatorname{dim}\left(\operatorname{ran} T_{-}\right)=\operatorname{dim} E_{T}(-\infty, 0) \mathfrak{H}$. If the form $\mathfrak{t}$ is closed and $T$ is the operator associated with it, $\mathfrak{t}=\mathfrak{t}_{T}$, (see [23]) then by virtue of the minimax principle $\varkappa_{-}(\mathfrak{t})=\varkappa_{-}(T)$.

Next we define the classes $C\left(\pi / 2 ; \varkappa_{ \pm}\right)$and $T \in C\left(\pi / 2 ; \mathfrak{S}^{ \pm}\right)$.

Definition 4.2. Let $\varkappa \in \mathbb{Z}_{+}$and $\mathfrak{S}$ be a two-sided ideal in $[\mathfrak{H}]$. We write

(a) $T \in C(\pi / 2 ; \varkappa)$ if $T \in\left[\mathfrak{H}, \mathfrak{H}^{\prime}\right]$ and $\varkappa_{-}\left(I-T^{*} T\right)=\varkappa$;

(b) $T \in C(\pi / 2 ; \mathfrak{S})$ if $T \in\left[\mathfrak{H}, \mathfrak{H}^{\prime}\right]$ and $\left(I-T^{*} T\right)_{-} \in \mathfrak{S}$.

Definition 4.3. Let $\varphi \in[0, \pi / 2]$, $\varkappa^{ \pm} \in \mathbb{Z}^{+}$, and let $\mathfrak{S}^{ \pm}$be two-sided ideals in $[\mathfrak{H}]$. An operator $T(\in[\mathfrak{H}])$ is put

(a) in the class $C_{\mathfrak{H}}\left(\varphi ; \varkappa^{ \pm}\right)$with $\varphi \in(0, \pi / 2]$, if

$$
T \sin \varphi \pm i \cos \varphi \cdot I \in C_{\mathfrak{H}}\left(\pi / 2 ; \varkappa^{ \pm}\right)
$$

(b) in the class $C_{\mathfrak{H}}\left(\varphi ; \mathfrak{S}^{ \pm}\right)$with $\varphi \in(0, \pi / 2]$, if

$$
T \sin \varphi \pm i \cos \varphi \cdot I \in C_{\mathfrak{H}}\left(\pi / 2 ; \mathfrak{S}^{ \pm}\right) .
$$

(c) in the class $C_{\mathfrak{H}}(0 ; \varkappa) \quad\left(C_{\mathfrak{H}}(0 ; \mathfrak{S})\right)$, if $T=T^{*}$ and $\varkappa_{-}(T)=\varkappa \quad\left(T_{-} \in \mathfrak{S}\right)$.

We write $C_{\mathfrak{H}}(\varphi ; \varkappa)$ and $C_{\mathfrak{H}}(\varphi ; \mathfrak{S})$ in place of $C_{\mathfrak{H}}\left(\varphi ; \varkappa^{ \pm}\right)$and $C_{\mathfrak{H}}\left(\varphi ; \mathfrak{S}^{ \pm}\right)$respectively if $\varkappa:=\varkappa^{+}=\varkappa^{-}$and $\mathfrak{S}:=\mathfrak{S}^{+}=\mathfrak{S}^{-}$;

Observe that the class $C_{\mathfrak{H}}\left(\pi / 2 ; \varkappa^{ \pm}\right)$is not empty only if $\varkappa^{+}=\varkappa^{-}$. Some properties of the class $C\left(\pi / 2 ; \mathfrak{S}_{\infty}\right)$ can be found in $[30$.

Lemma 4.4. [29, 21] Let $T_{1}=\left(\begin{array}{l}T_{11} \\ T_{21}\end{array}\right)\left(\in\left[\mathfrak{H}_{1}, \mathfrak{H}\right]\right)$ be a nonnegative symmetric operator $\left(\Longleftrightarrow T_{11} \geq\right.$ $0)$ admitting a bounded nonnegative selfadjoint extension and let $T(\in[\mathfrak{H}])$ be any selfadjoint extension of $T_{1}$ with the block-matrix representation $T=T^{*}=\left(T_{i j}\right)_{i, j=1}^{2}$ with respect to the orthogonal decomposition $\mathfrak{H}=\mathfrak{H}_{1} \oplus \mathfrak{H}_{2}$. Then

(i) $\Re\left(T_{11}^{1 / 2}\right) \supset \Re\left(T_{12}\right)$ and the operator $S:=T_{11}^{-1 / 2} T_{12}$ is well-defined and bounded;

(ii) $\varkappa_{-}(T)=\varkappa_{-}\left(T_{22}-S^{*} S\right)$. In particular, $T \geq 0$ iff $T_{22}-S^{*} S \geq 0$.

Now we are ready to present the corollaries.

Corollary 4.5. Let $\left\{T_{1}, T_{2}\right\}$ be a dual pair of symmetric contractions, $T_{K} \in \operatorname{Ext}_{\left\{T_{1}, T_{2}\right\}}$, $\varphi \in\left[\varphi_{1}, \pi / 2\right]$ and $\varphi_{1}>0$. Then the following equivalences are valid:

$$
T_{K} \in C_{\mathfrak{H}}\left(\varphi ; \varkappa^{ \pm}\right) \Longleftrightarrow K \sin \varphi \mp i Q \cos \varphi \in C\left(\pi / 2 ; \varkappa^{ \pm}\right)
$$

Proof. Let as in Theorem 4.1

$$
\left(G_{i j}^{ \pm}\right)_{i, j=1}^{2}:=G^{ \pm}:=I-T_{K}^{*} T_{K} \pm i \cot \varphi\left(T_{K}-T_{K}^{*}\right) .
$$


The operators $G_{0}^{ \pm}:=\left(\begin{array}{l}G_{11} \\ G_{21}^{ \pm}\end{array}\right)$are nonnegative $\left(\Longleftrightarrow G_{11} \geq 0\right)$. Moreover, both of them admit bounded nonnegative selfadjoint extensions. For example, the operator

$$
\left(\begin{array}{cc}
G_{11}^{1 / 2} & 0 \\
B_{+}^{*} & 0
\end{array}\right)\left(\begin{array}{cc}
G_{11}^{1 / 2} & B_{+} \\
0 & 0
\end{array}\right)=\left(\begin{array}{cc}
G_{11} & G_{12}^{+} \\
G_{21}^{+} & B_{+}^{*} B_{+}
\end{array}\right) \geq 0
$$

with a bounded $B_{+}=G_{11}^{-1 / 2} G_{12}^{+}$is a nonnegative extension of $G_{0}^{+}$. Therefore combining Lemma 4.4 with Theorem 4.1 (see equality (4.5)) we get

$$
\varkappa_{-}\left(I-T_{K}^{*} T_{K}\right)=\varkappa_{-}\left(G_{22}^{ \pm}-\left(G_{11}^{-1 / 2} G_{12}^{ \pm}\right)^{*}\left(G_{11}^{-1 / 2} G_{12}^{ \pm}\right)\right)=\varkappa_{-}\left(I-K_{\mp}^{*} K_{\mp}\right),
$$

where $K_{ \pm}:=K \sin \varphi \pm i Q \cos \varphi$.

Corollary 4.6. Let $\left\{T_{1}, T_{2}\right\}$ be a dual pair of symmetric contractions and $0 \in \rho\left(D_{T_{11}}\right) \cap$ $\rho\left(D_{V}\right)$. Suppose additionally that $\varphi \in\left[\varphi_{1}, \pi / 2\right]$ and $\varphi_{1}>0$. Then the following implications hold

$$
K \sin \varphi \pm Q \cos \varphi \in C\left(\pi / 2 ; \mathfrak{S}^{ \pm}\right) \Longrightarrow T_{K} \in C_{\mathfrak{H}}\left(\varphi ; \mathfrak{S}^{ \pm}\right)
$$

If additionally $0 \in \rho\left(D_{U}\right)$ then implications (4.37) turns into the equivalences.

Proof. The required assertion immediately follows from (4.5) and the identity

$$
\left(\begin{array}{cc}
I & 0 \\
-G_{21}^{ \pm} G_{11}^{-1} & I
\end{array}\right)\left(\begin{array}{cc}
G_{11} & G_{12}^{ \pm} \\
G_{21}^{ \pm} & G_{22}^{ \pm}
\end{array}\right)\left(\begin{array}{cc}
I & -G_{11}^{-1} G_{12}^{ \pm} \\
0 & I
\end{array}\right)=\left(\begin{array}{cc}
G_{11} & 0 \\
0 & G_{22}^{ \pm}-G_{21}^{ \pm} G_{11}^{-1} G_{12}^{ \pm}
\end{array}\right)
$$

Remark 4.7. (i) Let $\varphi=\pi / 2$. Then both relations (4.36) and (4.37) are simplified and take the form

$$
\begin{gathered}
K \in C(\pi / 2 ; \varkappa) \Longleftrightarrow T_{K} \in C(\pi / 2 ; \varkappa), \\
\left(I-K^{*} K\right)_{-} \in \mathfrak{S} \Longrightarrow\left(I-T_{K}^{*} T_{K}\right)_{-} \in \mathfrak{S} .
\end{gathered}
$$

Both relations have been established in [28, 30, for any (not necessary symmetric) dual pair of contractions.

(ii) Let $\varphi_{1}=\arccos \left(\left\|Q_{0}\right\|^{-1}\right)=0$. Then $\left\|Q_{0}\right\|=1$ and by Remark 3.5 (see (3.24) $) U=V^{*}$, that is $Q=I$ and $T_{1}=T_{2}$. In this case description of the sets $\operatorname{Ext}_{T_{1}}(\varphi ; \varkappa)=\operatorname{Ext}_{\left\{T_{1}, T_{1}\right\}}(\varphi ; \varkappa)$ and $\operatorname{Ext}_{T_{1}}(\varphi ; \mathfrak{S})=\operatorname{Ext}_{\left\{T_{1}, T_{1}\right\}}(\varphi ; \mathfrak{S}), \quad \varphi \in[0, \pi / 2]$, can easily be derived from Corollaries 4.5 and 4.6. Now in place of relations (4.36) and (4.37) we have

$$
K \in C_{\mathcal{H}}\left(\varphi ; \varkappa^{ \pm}\right) \Longleftrightarrow T_{K} \in C_{\mathfrak{H}}\left(\varphi ; \varkappa^{ \pm}\right)
$$

where $\mathcal{H}=\overline{\mathrm{ran}} D_{U}=\overline{\mathrm{ran}} D_{V^{*}}$. Both formulas have earlier been obtained in [28, 30]. Note also, that if $\varkappa^{ \pm}=0$ then formula (4.40) gives one more proof of Corollary [3.11. 
Definition 4.8. Let $\varphi \in[0, \pi / 2), \varkappa^{+} \in \mathbb{Z}_{+}, \mathfrak{S}^{ \pm}$two-sided ideals in $[\mathfrak{H}]$, and $B \in \mathcal{C}(\mathfrak{H})$. Let further, the quadratic forms

$$
\mathfrak{t}_{ \pm}[f]=\operatorname{Re}(B f, f) \pm \cot \varphi \cdot \operatorname{Im}(B f, f), \quad f \in \operatorname{dom} B,
$$

be semibounded below and $B^{ \pm}$the linear operators associated with their closures (the closability of the form $\mathfrak{t}_{ \pm}$is a consequence of their semiboundness (see [23])). We write

(a) $B \in S_{\mathfrak{H}}\left(\varphi ; \varkappa^{ \pm}\right)$, if $\rho(B) \neq \varnothing$ and $\varkappa\left(B^{ \pm}\right)=\varkappa^{ \pm}$;

(b) $B \in S_{\mathfrak{H}}\left(\varphi ; \mathfrak{S}^{ \pm}\right)$, if $\left(B^{ \pm}\right)_{-} \in \mathfrak{S}^{ \pm}$and $\rho(B) \neq \varnothing$.

$A$ closed linear relation $\theta$ in $\mathfrak{H}$ is also put in the class $S_{\mathfrak{H}}\left(\varphi ; \varkappa^{ \pm}\right)\left(S_{\mathfrak{H}}\left(\varphi ; \mathfrak{S}^{ \pm}\right)\right)$if $\operatorname{Re}\left(f^{\prime}, f\right) \geq$ $\beta\|f\|^{2}$ for all $\left\{f, f^{\prime}\right\} \in \theta$ (with some $\beta \in \mathbb{R}$ ) and its operator part is in $S_{\mathfrak{H}}\left(\varphi ; \varkappa^{ \pm}\right)\left(S_{\mathfrak{H}}\left(\varphi ; \mathfrak{S}^{ \pm}\right)\right)$.

It is clear that $S_{\mathfrak{H}}(\varphi ; 0)$ coinsides with $S_{\mathfrak{H}}(\varphi)$.

It is clear that the classes $C_{\mathfrak{H}}\left(\varphi ; \varkappa^{ \pm}\right)$and $S_{\mathfrak{H}}\left(\varphi ; \varkappa^{ \pm}\right)$are connected by means of the linear fractional transformation (2.14). The same is also true for the classes $C_{\mathfrak{H}}\left(\varphi ; \mathfrak{S}^{ \pm}\right)$and $S_{\mathfrak{H}}\left(\varphi ; \mathfrak{S}^{ \pm}\right)$.

4.3. Shorted operators. Here we present two additional corollaries from Theorem 4.1 complementing Theorem 3.1. For this purpose we recall some well-known results and the definition of a shorted operator.

Definition 4.9. (25]) For any nonnegative operator $A(\in[\mathfrak{H}])$ and a subspace $\mathfrak{N}(\subset \mathfrak{H})$ there exists the largest element in the set of all bounded operators not exceeding $A$ and annihilating $\mathfrak{N}^{\perp}=\mathfrak{H} \ominus \mathfrak{N}$. This element, is denoted by $A_{\mathfrak{N}}$ and is called the shorted to $\mathfrak{N}$ operator.

The transformation $A \rightarrow A_{\mathfrak{N}}$ is called the Krein transformation.

Lemma 4.10. (25, 37, 21]). Let $A=\left(A_{i j}\right)_{i, j=1}^{2}$ be a block-matrix representation of an operator $A \geq 0(A \in[\mathfrak{H}])$ with respect to the decomposition $\mathfrak{H}=\mathfrak{H}_{1} \oplus \mathfrak{N}$. Then the shorted to $\mathfrak{N}$ operator $A_{\mathfrak{N}}$ is of the form

$$
A_{\mathfrak{N}}=\left(\begin{array}{cc}
0 & 0 \\
0 & A_{22}-S^{*} S
\end{array}\right), \quad S=A_{11}^{-1 / 2} A_{12}
$$

If in addition $0 \in \rho\left(A_{11}\right)$ then $S^{*} S=A_{21} A_{11}^{-1} A_{12}$.

Corollary 4.11. (25, 26, 37]). Let $\mathfrak{H}=\mathfrak{H}_{1} \oplus \mathfrak{N}, A \in[\mathfrak{H}]$ and $A \geq 0$. Then

$$
\inf _{g \in \mathfrak{H}_{1}}(A(f-g), f-g)=\left(A_{\mathfrak{N}} f, f\right), \quad f \in \mathfrak{H} .
$$

Corollary 4.12. Let $\left\{T_{1}, T_{2}\right\}$ be a dual pair of symmetric contractions in $\mathfrak{H}=\mathfrak{H}_{1} \oplus \mathfrak{H}_{2}$, $\varphi \in\left[\varphi_{1}, \pi / 2\right]$ where $\varphi_{1}:=\arccos \left(\left\|Q_{0}\right\|^{-1}\right)$ and $T_{K} \in \operatorname{Ext}_{\left\{T_{1}, T_{2}\right\}}(\varphi)$. Then the shorted to $\mathfrak{N}:=\mathfrak{H}_{2}$ operators

$$
G^{ \pm}=I-T_{K}^{*} T_{K} \pm i \cot \varphi\left(T_{K}-T_{K}^{*}\right) \quad \text { and } \quad S^{ \pm}=I-T_{K} T_{K}^{*} \pm i \cot \varphi\left(T_{K}-T_{K}^{*}\right)
$$

have the following form

$$
\left(G^{ \pm}\right)_{\mathfrak{N}}=\left(\begin{array}{cc}
0 & 0 \\
0 & \sin ^{-2} \varphi \cdot D_{U}\left[I-\left(K^{*} \sin \varphi \pm i Q^{*} \cos \varphi\right)(K \sin \varphi \mp i Q \cos \varphi)\right] D_{U}
\end{array}\right)
$$

and

$$
\left(S^{ \pm}\right)_{\mathfrak{N}}=\left(\begin{array}{cc}
0 & 0 \\
0 & \sin ^{-2} \varphi \cdot D_{V^{*}}\left[I-(K \sin \varphi \mp i Q \cos \varphi)\left(K^{*} \sin \varphi \pm i Q^{*} \cos \varphi\right)\right] D_{V^{*}}
\end{array}\right)
$$

Proof. One deduces the proof combining Theorem 4.1 with Lemma 4.10. 
Corollary 4.13. Let $\left\{T_{1}, T_{2}\right\}$ be a dual pair of Hermitian contractions in $\mathfrak{H}=\mathfrak{H}_{1} \oplus \mathfrak{H}_{2}$, and $T_{K} \in \operatorname{Ext}_{\left\{T_{1}, T_{2}\right\}}(\varphi)$. Suppose additionally that $\varphi_{1}=\arccos \left(\left\|Q_{0}\right\|^{-1}\right)<\pi / 2, \quad \varphi \in\left[\varphi_{1}, \pi / 2\right]$ and $\mathfrak{S}^{ \pm}$are two-sided ideals in $[\mathfrak{H}]$. Then

(i) $\operatorname{Ext}_{\left\{T_{1}, T_{2}\right\}}(\varphi) \neq \emptyset$ if and only if $\varphi \in\left[\varphi_{1}, \pi / 2\right]$;

(ii) The following equivalence holds

$$
T_{K} \in \operatorname{Ext}_{\left\{T_{1}, T_{2}\right\}}(\varphi) \Longleftrightarrow K_{ \pm}:=K \sin \varphi \pm i Q \cos \varphi \in C(\pi / 2) ;
$$

(iii) The following implications hold with $\mathfrak{N}:=\mathfrak{H}_{2}$

$$
D_{K_{\mp}}^{2} \in \mathfrak{S}^{ \pm} \Longrightarrow\left(G^{ \pm}\right)_{\mathfrak{N}} \in \mathfrak{S}^{ \pm}, \quad D_{K_{\mp}^{*}}^{2} \in \mathfrak{S}^{ \pm} \Longrightarrow\left(S^{ \pm}\right)_{\mathfrak{N}} \in \mathfrak{S}^{ \pm} .
$$

If additionally $0 \in \rho\left(D_{U}\right)$ then implications (4.48) turn into the equiavalences.

Proof. (i)-(ii) By definition $T_{K} \in C_{\mathfrak{H}}(\varphi)$ if and only if $G^{ \pm} \in C_{\mathfrak{H}}(\pi / 2)$, where $G^{ \pm}$are defined by (4.44). Note that $G_{11}^{ \pm}=G_{11}=I-T_{11}^{*} T_{11}-T_{21}^{*} T_{21} \geq 0$ since $T_{1}$ is a contraction. Therefore by Sylvester criterion (see Lemma 4.4) $G^{ \pm} \geq 0$ iff $\left(G^{ \pm}\right)_{\mathfrak{N}} \geq 0$ with $\mathfrak{N}=\mathfrak{H}_{2}$. Combining this inequality with (4.45) we arrive at equivalence (4.47).

Hence, if $\operatorname{Ext}_{\left\{T_{1}, T_{2}\right\}}(\varphi) \neq \emptyset$ then $Q \cos \varphi \in C(\pi / 2)$, that is $\varphi \in\left[\varphi_{1}, \pi / 2\right]$. Conversly, if $\varphi \in\left[\varphi_{1}, \pi / 2\right]$ then $Q \cos \varphi \in C(\pi / 2)$ and the operator $T_{0}$, that is $T_{K}$ with $K=0$, belongs to $\operatorname{Ext}_{\left\{T_{1}, T_{2}\right\}}(\varphi)$.

(iii) This statement is immediately implied by formulas (4.45) and (4.46).

Remark 4.14. (i) Suppose that in Corollary $4.12 \varphi=\pi / 2$. Then $G^{ \pm}=I-T_{K}^{*} T_{K}=D_{T_{K}}^{2}$ and $S^{ \pm}=I-T_{K} T_{K}^{*}=D_{T_{K}^{*}}^{2}$. Now formulas (4.45) and (4.46) are simplified and take the form

$$
\left(D_{T_{K}}^{2}\right)_{\mathfrak{N}}=\left(\begin{array}{cc}
0 & 0 \\
0 & D_{U} D_{K}^{2} D_{U}
\end{array}\right), \quad\left(D_{T_{K}^{*}}^{2}\right)_{\mathfrak{N}^{\prime}}=\left(\begin{array}{cc}
0 & 0 \\
0 & D_{V^{*}} D_{K}^{2} D_{V^{*}}
\end{array}\right)
$$

where $\mathfrak{N}:=\mathfrak{H}_{2}$ and $\mathfrak{N}^{\prime}:=\mathfrak{H}_{2}^{\prime}$. Both formulas have earlier been obtained in [28, 30] for any (not necessary symmetric) dual pair of contractions.

(ii) Corollary 4.13 (iii) complements Theorem 3.4. Moreover, Corollary 4.13 gives another proof of Theorem [3.4. Indeed, in the case $0 \in \rho\left(D_{U}\right) \cap \rho\left(D_{V^{*}}\right)$ the proof of Theorem 4.1] does not depend on Theorems 3.4 and 3.14. The proof of equivalence (4.47) without the additional assumption $0 \in \rho\left(D_{U}\right) \cap \rho\left(D_{V^{*}}\right)$ can easily be obtained by considering the family $\left\{r T_{1}, r T_{2}\right\}, r \in(0,1)$, of dual pairs of contractions and passage to the limit as $r \rightarrow 1$ (cf. the proof of Theorem 3.14).

\section{COMPletions of A SPECial triangular OPERATOR-MATrix.}

5.1. A complement to the S. Nagy and C. Foias result. Here we describe the operators $T_{12}$ completing the incomplete contractive operator block-matrix

$$
\left(\begin{array}{cc}
T_{11} & * \\
0 & T_{22}
\end{array}\right)
$$

to form an operator matrix of some class.

We start with the following S. Nagy and C. Foias result. 
Proposition 5.1. 35] Let $\mathfrak{H}=\mathfrak{H}_{1} \oplus \mathfrak{H}_{2}=\mathfrak{H}_{1}^{\prime} \oplus \mathfrak{H}_{2}^{\prime}$ and let $T_{j j}\left(\in\left[\mathfrak{H}_{j}, \mathfrak{H}_{j}^{\prime}\right]\right)$ be a contraction, $j \in\{1,2\}$. Then the family of operators $T_{12} \in\left[\mathfrak{H}_{2}, \mathfrak{H}_{1}^{\prime}\right]$, completing the block-matrix (5.1) to a contractive matrix $T=\left(T_{i j}\right)_{i, j=1}^{2} \in[\mathfrak{H}]$, forms an operator ball $B\left(0 ; D_{T_{11}^{*}}, D_{T_{22}}\right)$, that is

$$
T=\left(\begin{array}{cc}
T_{11} & T_{12} \\
0 & T_{22}
\end{array}\right) \in C_{\mathfrak{H}}(\pi / 2) \Longleftrightarrow T_{12}=D_{T_{11}^{*}} K D_{T_{22}}, \quad\|K\| \leq 1, \quad K \in\left[\mathcal{H}_{2}, \mathcal{H}_{1}\right] .
$$

Proof. Let at first $0 \in \rho\left(D_{T_{22}}\right)$. Then according to the Sylvester criterion the equivalence

$$
I-T^{*} T \geq 0 \Longleftrightarrow D_{T_{22}}^{2}-Z^{*}\left(I+T_{11} D_{T_{11}}^{-2} T_{11}^{*}\right) Z \geq 0,
$$

holds true with $Z:=T_{12}$. By Lemma 2.3 the set of solutions of (5.2) forms an operator ball. Observing that $I+T_{11} D_{T_{11}}^{-2} T_{11}^{2}=D_{T_{11}^{*}}^{-2}$ and applying Lemma 2.3 to (5.2) we arrive at the required relation

$$
T_{12}=Z=D_{T_{11}^{*}} K D_{T_{22}}, \quad\|K\| \leq 1, \quad K \in\left[\mathcal{H}_{2}, \mathcal{H}_{1}\right]
$$

We may easily free ourselves of the condition $0 \in \rho\left(D_{T_{22}}\right)$ by virtue of passage to the limit.

Thus the contractive "completions" of the matrix (5.1) are of the form

$$
T_{K}=\left(\begin{array}{cc}
T_{11} & D_{T_{11}^{*}} K D_{T_{22}} \\
0 & T_{22}
\end{array}\right)
$$

with $\|K\| \leq 1, K \in\left[\mathcal{H}_{2} \cdot \mathcal{H}_{1}\right]$.

Let us now consider "completions" of the incomplete block-matrix (5.1) of the form (5.3) not assuming the operator $K$ to be a contraction.

Proposition 5.2. Let $\mathfrak{H}=\mathfrak{H}_{1} \oplus \mathfrak{H}_{2}=\mathfrak{H}^{\prime} \oplus \mathfrak{H}_{2}^{\prime}, T_{j j} \in\left[\mathfrak{H}_{j}, \mathfrak{H}_{j}^{\prime}\right]$, and $\left\|T_{j j}\right\| \leq 1, j \in\{1,2\}$. Assume that $T_{K}$ is an operator matrix of the form (5.3) with $K \in\left[\mathcal{H}_{2}, \mathcal{H}_{1}\right]$ and put $G:=$ $\left(G_{i j}\right)_{i, j=1}^{2}=I-T_{K}^{*} T_{K}$ and $S:=\left(S_{i j}\right)_{i, j=1}^{2}=I-T_{K} T_{K}^{*}$. Then

(i) $\operatorname{ran}\left(G_{12}\right) \subset \operatorname{ran}\left(G_{11}^{1 / 2}\right)$ and $\operatorname{ran}\left(S_{12}\right) \subset \operatorname{ran}\left(S_{11}^{1 / 2}\right)$, hence the operators $G_{11}^{-1 / 2} G_{12}$ and $S_{11}^{-1 / 2} S_{12}$ are well defined and bounded;

(ii) the following identities are valid:

$$
\begin{gathered}
G_{22}-\left(G_{11}^{-1 / 2} G_{12}\right)^{*}\left(G_{11}^{-1 / 2} G_{12}\right)=D_{T_{22}}\left(I-K^{*} K\right) D_{T_{22}} \\
S_{22}-\left(S_{11}^{-1 / 2} S_{12}\right)^{*}\left(S_{11}^{-1 / 2} S_{12}\right)=D_{T_{11}^{*}}\left(I-K K^{*}\right) D_{T_{11}^{*}}
\end{gathered}
$$

Proof. Imposing the condition $0 \in \rho\left(D_{T_{11}}\right)$ we have

$$
\begin{array}{r}
G_{22}-G_{12}^{*} G_{11}^{-1} G_{12} \\
=D_{T_{22}}\left(I-K^{*} D_{T_{11}^{*}} K\right) D_{T_{22}}-D_{T_{22}} K^{*} D_{T_{11}^{*}} T_{11} D_{T_{11}}^{-2} T_{11}^{*} D_{T_{11}^{*}} K D_{T_{22}} \\
=D_{T_{22}}\left(I-K^{*} K+K^{*} T_{11} T_{11}^{*} K-K^{*} T_{11} T_{11}^{*} K\right) D_{T_{22}}=D_{T_{22}} D_{K}^{2} D_{T_{22}} .
\end{array}
$$

We may free ourselves of the condition $0 \in \rho\left(D_{T_{11}}\right)$ by passage to the limit just like it was done in the proof of Theorem 3.14. Equality (5.5) may be proved similarly.

Corollary 5.3. Suppose that conditions of Proposition 5.2 are satisfied. Then

(i) the following equivalence holds

$$
K \in C(\pi / 2 ; \varkappa) \cap\left[\mathcal{H}_{2}, \mathcal{H}_{1}\right] \Longleftrightarrow T_{K} \in C_{\mathfrak{H}}(\pi / 2 ; \varkappa) ;
$$


(ii) if in addition $0 \in \rho\left(G_{11}\right)$, then for any two-sided ideal $\mathfrak{S}$ in $[\mathfrak{H}]$ the following implication holds

$$
K \in C(\pi / 2 ; \mathfrak{S}) \Longrightarrow T_{K} \in C_{\mathfrak{H}}(\pi / 2 ; \mathfrak{S}) .
$$

This implication turns into the equivalence if additionally $0 \in \rho\left(D_{T_{22}}\right)$.

Corollary 5.4. Let $T_{K}$ be a contraction of the form (5.3). Then the operators $G:=D_{T_{K}}^{2}$ and $S:=D_{T_{K}^{*}}^{2}$ shorted to $\mathfrak{N}:=\mathfrak{H}_{2}$ and $\mathfrak{N}^{\prime}:=\mathfrak{H}_{2}^{\prime}$ respectively have the form

$$
G_{\mathfrak{N}}=\left(\begin{array}{cc}
0 & 0 \\
0 & D_{T_{22}} D_{K}^{2} D_{T_{22}}
\end{array}\right) \quad \text { and } \quad S_{\mathfrak{N}^{\prime}}=\left(\begin{array}{cc}
0 & 0 \\
0 & D_{T_{11}^{*}} D_{K^{*}}^{2} D_{T_{11}^{*}}
\end{array}\right) .
$$

Corollaries 5.3 and 5.4 may be derived from Proposition 5.2 just like Corollaries 4.5, 4.6 and 4.12 from Theorem 4.1 .

\subsection{A solution to Yu. L. Shmul'yan's problem.}

The following proposition provides an answer to the Yu. L. Shmul'yan question.

Proposition 5.5. Let $\mathfrak{H}=\mathfrak{H}_{1} \oplus \mathfrak{H}_{2}, T_{j j} \in C_{\mathfrak{H}_{j}}(\varphi), j \in\{1,2\}$, and $\varphi \in(0, \pi / 2)$. Then

(i) there exist contractions $U_{\varphi}=U_{\varphi}^{*} \in C_{\mathfrak{H}_{1}}(\pi / 2)$ and $V_{\varphi}=V_{\varphi}^{*} \in C_{\mathfrak{H}_{2}}(\pi / 2)$ such that

$$
2 \cot \varphi\left(\operatorname{Im} T_{11}\right)=D_{T_{11}^{*}} U_{\varphi} D_{T_{11}^{*}} \quad \text { and } \quad 2 \cot \varphi\left(\operatorname{Im} T_{22}\right)=D_{T_{22}} V_{\varphi} D_{T_{22}} ;
$$

(ii) the following equivalence holds true

$$
\begin{array}{r}
T=\left(\begin{array}{cc}
T_{11} & T_{12} \\
0 & T_{22}
\end{array}\right) \in C_{\mathfrak{H}}(\varphi) \Longleftrightarrow \\
T_{12}=\sin \varphi D_{T_{11}^{*}} K D_{T_{22}}, \quad\left(I \pm U_{\varphi}\right)^{-1 / 2} K\left(I \pm V_{\varphi}\right)^{-1 / 2} \in C(\pi / 2), \quad K \in\left[\mathcal{H}_{2}, \mathcal{H}_{1}\right] .
\end{array}
$$

Proof. (i) Equalities (5.6) have already been proved in Proposition 3.8 .

(ii) The inclusion $T \in C_{\mathfrak{H}}(\varphi)$ means that $T \sin \varphi \pm i \cos \varphi \cdot I \in C_{\mathfrak{H}}(\pi / 2)$. Since $T_{j j} \sin \varphi \pm$ $i \cos \varphi \cdot I \in C_{\mathfrak{H}_{j}}(\pi / 2), j \in\{1,2\}$, then by Proposition 5.1 the equivalences

$$
T \sin \varphi \pm i \cos \varphi \cdot I \in C(\pi / 2) \Longleftrightarrow T_{12} \sin \varphi=R_{l}^{ \pm} K_{ \pm} R_{r}^{ \pm}, \quad\left\|K_{ \pm}\right\| \leq 1,
$$

hold true. Here $K_{ \pm} \in\left[\mathcal{H}_{r}^{ \pm}, \mathcal{H}_{l}^{ \pm}\right], \mathcal{H}_{r}^{ \pm}=\overline{\operatorname{ran}}\left(R_{r}^{ \pm}\right), \mathcal{H}_{l}^{ \pm}=\overline{\operatorname{ran}}\left(R_{l}^{ \pm}\right)$, and the operators $R_{l}^{ \pm}$ and $R_{r}^{ \pm}$are defined by

$$
\begin{aligned}
& \left(R_{l}^{ \pm}\right)^{2}=\sin ^{2} \varphi D_{T_{11}^{*}}^{2} \pm \sin 2 \varphi \cdot\left(\operatorname{Im} T_{11}\right)=\sin ^{2} \varphi \cdot D_{T_{11}^{*}}\left(I \pm U_{\varphi}\right) D_{T_{11}^{*}} \\
& \left(R_{r}^{ \pm}\right)^{2}=\sin ^{2} \varphi D_{T_{22}}^{2} \pm \sin 2 \varphi \cdot\left(\operatorname{Im} T_{22}\right)=\sin ^{2} \varphi \cdot D_{T_{22}}\left(I \pm V_{\varphi}\right) D_{T_{22}} .
\end{aligned}
$$

It is clear that

$$
R_{l}^{2}:=\left(R_{l}^{+}\right)^{2}+\left(R_{l}^{-}\right)^{2}=2 \sin ^{2} \varphi D_{T_{11}^{*}}, \quad R_{r}^{2}:=\left(R_{r}^{+}\right)^{2}+\left(R_{r}^{-}\right)^{2}=2 \sin ^{2} \varphi D_{T_{22}}^{2} .
$$

Further, relations (5.9) yield polar representations for the operators $\sin \varphi \cdot\left(I \pm U_{\varphi}\right)^{1 / 2} D_{T_{11}^{*}}$ and $\sin \varphi \cdot\left(I \pm V_{\varphi}\right)^{1 / 2} D_{T_{22}}$. Namely, we have

$$
\sin \varphi \cdot\left(I \pm U_{\varphi}\right)^{1 / 2} D_{T_{11}^{*}}=U_{ \pm} R_{l}^{ \pm} \quad \text { and } \quad \sin \varphi \cdot\left(I \pm V_{\varphi}\right)^{1 / 2} D_{T_{22}}=V_{ \pm} R_{r}^{ \pm}
$$

where $U_{ \pm}$and $V_{ \pm}$are partial isometries with initial spaces $\mathcal{H}_{l}^{ \pm}$and $\mathcal{H}_{r}^{ \pm}$respectively. We deduce the following equalities from (5.10) and (5.11):

$$
R_{l}^{ \pm}=\frac{1}{\sqrt{2}} R_{l}\left(I \pm U_{\varphi}\right)^{1 / 2} U_{ \pm}, \quad R_{r}^{ \pm}=\frac{1}{\sqrt{2}} V_{ \pm}^{*}\left(I \pm V_{\varphi}\right)^{1 / 2} R_{r} .
$$


Taking (5.12) into account we rewrite expression (5.8) for $T_{12} \sin \varphi$ in the form

$$
T_{12} \sin \varphi=\frac{1}{2} R_{l}\left(I \pm U_{\varphi}\right)^{1 / 2} U_{ \pm} K_{ \pm} V_{ \pm}^{*}\left(I \pm V_{\varphi}\right)^{1 / 2} R_{r}
$$

It follows from (5.13) that

$$
\left(I+U_{\varphi}\right)^{1 / 2} U_{+} K_{+} V_{+}^{*}\left(I+V_{\varphi}\right)^{1 / 2}=\left(I-U_{\varphi}\right)^{1 / 2} U_{-} K_{-} V_{-}^{*}\left(I-V_{\varphi}\right)^{1 / 2} .
$$

Denoting the operator in the left-hand side of (5.14) by $K$ and taking into account (5.10) we arrive at the following formula for $T_{12}$ :

$$
T_{12}=\frac{1}{2 \sin \varphi} R_{l} K R_{r}=\sin \varphi D_{T_{11}^{*}} K D_{T_{22}},
$$

with $\left(I \pm U_{\varphi}\right)^{-1 / 2} K\left(I \pm V_{\varphi}\right)^{-1 / 2} \in C(\pi / 2)$. Here we have made use of the obvious equivalences $U_{ \pm} K_{ \pm} V_{ \pm}^{*} \in C(\pi / 2) \Longleftrightarrow K_{ \pm} \in C(\pi / 2)$.

\section{REFERENCES}

[1] N.I. Akhiezer and I.M. Glazman, Theory of linear operators in Hilbert space, Moscow "Nauka", 1966.

[2] A. Alonso and B. Simon, "The Birman-Kreln-Vishik theory of self-adjoint extensions of semibounded operators", J. Operator Theory, 4 (1980), 251-270.

[3] T. Ando and K. Nishio, "Positive selfadjoint extensions of positive symmetric operators", Tôhoku Math. J., 22 (1970), 65-75.

[4] Yu. M. Arlinskii, On a class of nondensely defined contractions and their extensions, J. of Math. Sci., v.97, No 5 (1999), 4391-4419.

[5] Yu. M. Arlinskiı̆ and E. R. Tsekanovskiŭ, Maximal sectorial extensions of positive Hermitian operators and their resolvents, Dokl. Acad. Nauk Armyan. SSR, 79, No. 5 (1984), 199-202.

[6] Yu. M. Arlinskiǔ and E. R. Tsekanovskiü, Quasiself-adjoint contractive extensions of Hermitian contractions, Teor. Funkts., Funksional. Anal. i Prilozen., 50 (1988), 9-16.

[7] Gr. Arsene and A. Gheondea, "Completing matrix contractions", J. Operator Theory, 7 (1982), $179-189$.

[8] Gr. Arsene, T. Constantinescu and A. Gheondea, Lifting of operators and prescribed numbers of negative sguares, Michigan Math. J., 34 (1987), 201-216.

[9] M.S. Birman, "On the self-adjoint extensions of positive definite operators", Mat. Sb., 38 (1956), 431450 .

[10] J.F. Brasche and H. Neidhardt, "Some remarks on Krein's extension theory", Math. Nachr., 165 (1994), 159-181.

[11] Ch. Davis, Some dilation representation Theorems, in the book Proc.of the Second Intern. Symp. in West Africa on funct. anal. and its appl.-Kunasi, 1979, 159-182.

[12] Ch. Davis, W. M. Kahan and H. F. Weinberger, Norm-preserving dilations and their applications to optimal error bounds, Siam J. Numerical Anal., 19, No. 3, (1982), 445-469.

[13] V.A. Derkach and M.M. Malamud, "Generalized resolvents and the boundary value problems for Hermitian operators with gaps", J. Funct. Anal., 95 (1991), 1-95.

[14] V.A. Derkach and M.M. Malamud, "The extension theory of Hermitian operators and the moment problem", J. Math. Sciences, 73 (1995), 141-242.

[15] V.A. Derkach, M.M. Malamud and E.R. Tsekanovskii, "Sectorial extensions of positive operators and characteristic functions", Dokl. Acad. Sci. USSR, 298, No 3 (1988), 537-541.

[16] P.A. Fillmore and J.P. Williams, "On operator ranges", Adv. Math., 7 (1971), 254-281.

[17] C. Foias and A.E. Frazho, "Redheffer products and the lifting of contractions on Hilbert space", J. Operator theory, 11 (1984), 193-196.

[18] I. M. Glazman and Yu. I. Lyubitch, Finite dimensional Linear Analysis, Moscow, Nauka, 1969.

[19] Gorbachuk M.L. and Gorbachuk V.I., Boundary Value Problems for Operator Differential Equations, Kluver Academic Publishers, Dordrecht, 1991. 
[20] P.R. Halmos, A Hilbert space problem book, Princeton, New Jersey, 1967.

[21] S. Hassi, M.M. Malamud, and H.S.V. de Snoo, "On Krein's extension theory of nonnegative operators", Math. Nachr. 274-275 (2004), 40-73.

[22] K.O. Friedrichs, "Spektraltheorie halbbeschränkter Operatoren und Anwendung auf die Spektralzerlegung von Differentialoperatoren", Math. Ann., 109 (1934), 465-487.

[23] T. Kato, Perturbation theory for linear operators, Springer Verlag, Berlin, 1966.

[24] V.U. Kolmanovich and M.M. Malamud, "Extensions of Sectorial operators and dual pair of contractions", (Russian) Manuscript No 4428-85. Deposited at Vses. Nauchn-Issled, Inst. Nauchno-Techn. Informatsii, VINITI 1904 85, Moscow, R ZH Mat 10B1144, (1985), 1-57.

[25] M. G. Krein, "The theory of selfadjoint extensions of semibounded Hermitian operators and its applications, I", Mat. Sb., 20 (1947), 431-495.

[26] M. G. Krel̆n and I.E. Ovcharenko, "On the $Q$-functions and sc-resolvents of a nondensely defined Hermitian contraction", Sib. Math. J., 18 No. 5 (1977), 1032-1056.

[27] S. G. Krein Linear Differential Eguations in a Banach Space, Amer. Math. Soc., Providence, Rhode Island, 1971.

[28] M.M. Malamud, "On extensions of Hermitian and sectorial operators and dual pairs of contractions", Sov. Math. Dokl., 39, No. 2 (1989), 253-259.

[29] M.M. Malamud, "Certain classes of extensions of a lacunary Hermitian operator", Ukrainian Math. Journ., 44 No. 2 (1992), 190-204.

[30] M.M. Malamud, "On some classes of extensions of a sectorial operators and dual pairs of contractions" , Operator Theory: Adv. and Appl., Vol. 124 (2001), 401-449.

[31] S. Parrot, "On a quotient norm and the Sz.-Nagy-Foias Lifting Theorem", J. Funct. Anal., 30 (1978), 311-328.

[32] R. S. Phillips, "Dissipative operators and hyperbolic systems of partial differential eguations", Trans. Amer. Math. Soc., 90 (1959), 192-254.

[33] R. S. Phillips, "The extension of dual subspaces invariant under an algebra" (in the book Proc. Inter. Symp. Linear Algebra, Israel, 1960, Academic Press) (1961), 366-398.

[34] F. Riesz and B. Sz.-Nagy, Lecons D'Aanalyse Fonctionnelle, Akademiai Kiado, Budapest, 1972.

[35] B. Sz.-Nagy and C. Foias, Forme triangulaire d'un contraction et factorization de la fonction caracteristigue, Acta Sci. Math. (Szeged), 28 (1967), 201-212.

[36] B. Sz.-Nagy and C. Foias, Harmonic analysis of operators on Hilbert space, Amsterdam - Budapest, 1970.

[37] Yu. L. Shmul'yan, "A Hellinger operator integral", Mat. Sb., 49 (91), (1959), 381-430.

[38] Yu. L. Shmul'yan and R. N. Yanovskaya, "On matrices whose entries are contractions", Izv. Vissh. Ucheb. Zaved. Matematica, 7 (230), (1981), 72-75.

[39] M.I. Vishik, "On general boundary problems for elliptic differential equations", Trans. Moscow Math. Soc., 1 (1952), 186-246. (Russian) [English translation: Amer. Math. Soc. Transl., 24 (1963), 107-172].

Department of Mathematics, Donetsk National University, Universitetskaya str. 24, 83055 DONETSK, UKRAINE

E-mail address: mdm@dc.donetsk.ua 